Sharif University of Technology
Scientia Iranica
Transactions E: Industrial Engineering
SCIENTIA
I RAN I C A

\title{
Two-tuple linguistic aggregation operators based on subjective sensation and objective numerical scales for multi-criteria group decision-making problems
}

\author{
Y.-X. Ma, J. Wang, J.-Q. Wang* and X.-H. Chen \\ School of Business, Central South University, Changsha 410083, PR China.
}

Received 17 September 2014; received in revised form 24 December 2014; accepted 25 July 2015

\author{
KEYWORDS \\ Multi-criteria group \\ decision-making; \\ 2-tuple; \\ Subjective sensation \\ scale; \\ Objective numerical \\ scale; \\ Aggregation \\ operators.
}

\begin{abstract}
Many 2-tuple linguistic aggregation operators and linguistic Multi-Criteria Group Decision-Making (MCGDM) approaches have been successfully applied to numerous problems; they are difficult to reflect the different semantics of linguistic terms, distances between adjacent linguistic terms, and the subjective sensations of decision-makers in diverse decision-making problems. In this paper, some 2-tuple linguistic aggregation operators are proposed which are based on the subjective sensation scale and objective numerical scale, and a method is developed, which is based on the proposed operators, to overcome the aforementioned limitations. Firstly, the subjective sensation scale based on linguistic term sets and the subjective sensation scale and objective numerical scale based on 2-tuples are presented. Then, some 2-tuple linguistic operators based on the two scales are developed, namely the Generated Extended 2-Tuple Weighted Averaging (GE2T-WA) operator, Generated Extended 2-Tuple Ordered Weighted Averaging (GE2T-OWA) operator, Generated Extended 2-Tuple Weighted Geometric (GE2TWG) operator, and Generated Extended 2-Tuple Ordered Weighted Geometric (GE2TOWG) operator. Subsequently, based on the GE2T-WA and GE2T-OWA operators, or on the GE2T-WG and GE2T-OWA operators, an MCGDM method is developed. Finally, an example is provided and the proposed method is compared with some existing approaches, using the same illustrative example, for confirming its feasibility and rationality.
\end{abstract}

(C) 2016 Sharif University of Technology. All rights reserved.

\section{Introduction}

Studies on Multi-Criteria Group Decision-Making (MCGDM) problems that investigate a wide range of practical issues have made a great deal of progress in recent years and many MCGDM methods that use quantitative data have been proposed for solving such problems. Owing to the complexity of the objects and the vagueness of human thinking, it is difficult to obtain

\footnotetext{
*. Corresponding author. Tel.: +8673188830594; Fax: +867318710006

E-mail address: jqwang@csu.edu.cn (J.-Q. Wang)
}

accurate assessment values when evaluating objects without incurring high costs. Therefore, decisionmakers often use linguistic descriptors to express their assessments when facing those difficulties and these decision-making cases are linguistic MCGDM problems. The existing approaches for solving linguistic MCGDM problems include the following four types: converting linguistic assessment information into fuzzy numbers [1-5]; using the index of linguistic terms to resolve linguistic assessment information, and assemble the alternatives with the defined aggregation operators or outranking relations [6-11]; applying the cloud model [12-15]; and relying on the 2-tuple linguistic 
representation model for computing with words to solve MCGDM problems [16-20]. The 2-tuple linguistic models can overcome the shortcomings of information loss and distortion that may exist in converting linguistic assessment information into fuzzy numbers; moreover, they also can guarantee the continuity of linguistic assessment information, which can make the result of linguistic assessment information practical and understandable.

There has been a large amount of progress in research on investigating 2-tuple linguistic models for computing with words in decision-making since the 2tuple linguistic representation model was introduced by Herrera and Martinez [21]. In order to overcome the limitation of Herrera and Martinez's model [21], which is only suitable for the linguistic term sets that are uniformly and symmetrically distributed, some 2tuple linguistic models have been proposed for dealing with more complex linguistic information that includes non-homogeneous information [22,23], multi-granular linguistic information [24-27], and unbalanced linguistic information [28-31]. These models can improve the accuracy and recognition of the processing results in decision-making. Moreover, some extended 2-tuple linguistic models have been proposed for extending the model to improve the feasibility and accuracy of computing with words. Wang and Hao [32] extended the original model [21] by presenting a proportional 2-tuple fuzzy linguistic representation model and $\mathrm{Li}$ and Dong [33] developed an MCGDM method with linguistic assessments and linguistic weights based on this model. However, the semantics of linguistic terms in the linguistic term set used in the models [32] can only be defined by using symmetrical trapezoidal fuzzy numbers [34]. Dong et al. [34] generalized their proposals by considering linguistic 2-tuples under more general contexts and extended the 2-tuple fuzzy linguistic representation models by using the numerical scale for transforming the linguistic 2-tuples into numerical values. Due to the fact that the numerical scale model can provide a unified framework to integrate the models of Herrera and Martinez [21], Wang and Hao [32], and Herrera et al. [28], the interval version of the 2-tuple fuzzy linguistic representation model, which was based on the interval numerical scale $[35,36]$, and models that apply the numerical scale to unbalanced linguistic information [37] and the analytic hierarchy process [38] have been proposed for effectively improving precision of the results in computing with words.

In light of the fact that information aggregation always plays an important role in the 2-tuple linguistic decision-making process, many 2-tuple linguistic aggregation operators have been proposed to aggregate linguistic information. These operators generally include the following eight cases: (1) the
2-Tuple Arithmetic Averaging (TAA) operator, 2Tuple Weighted Averaging (TWA) operator, 2-Tuple Ordered Weighted Averaging (TOWA) operator, Extended 2-Tuple Weighted Averaging (ET-WA) operator developed by Herrera and Martinez [21] and some researchers extended 2-tuple linguistic operators based on these four operators $[17,18,39-42]$; (2) the 2-Tuple Linguistic Geometric Bonferroni Mean (2TLGBM) operator and Weighted 2-Tuple Linguistic Geometric Bonferroni Mean (W2TLGBM) operator [43]; (3) the 2-Tuple Linguistic Weighted Harmonic Averaging (TWHA) operator, 2-Tuple Linguistic Ordered Weighted Harmonic Averaging (TOWHA) operator, 2Tuple Linguistic Combined Weighted Harmonic Averaging (TCWHA) operator [44], and the extended 2tuple linguistic harmonic operators [45]; (4) the 2-Tuple Hybrid Weighted Arithmetic Average (THWA) operator, 2-Tuple Hybrid Linguistic Weighted Arithmetic Average (T-HLWA) operator, and Extended 2-Tuple Hybrid Linguistic Weighted Arithmetic Average (ETHLWA) operator [46]; (5) the Proportional 2-Tuple Weighted Geometric Averaging (PTWGA) operator, Proportional 2-Tuple Ordered Weighted Geometric Averaging (PTOWGA) operator, and Proportional 2Tuple Hybrid Geometric Averaging (PTHGA) operator [47]; (6) 2-Tuple Linguistic Power Average (2TLPA) operator, 2-Tuple Linguistic Power Weighted Average (2TLPWA) operator, 2-Tuple Linguistic Power Ordered Weighted Average (2TLPOWA) operator [47], and the Linguistic Proportional 2-Tuple Power Average (LP2TPA) operator [29]; (7) the Dependent Interval 2-Tuple Weighted Average (DITWA) operator, and Dependent Interval 2-Tuple Weighted Geometric (DITWG) operator [49]; (8) the interval 2-tuple correlated averaging operator and the interval 2-tuple correlated geometric operator [50]. However, when using most of the operators mentioned above, the distances between adjacent linguistic terms given by decision-makers are assumed to be equal, and the linguistic terms symmetrically distributed, as occurs when using the operators proposed in $[39,40]$. Such an assumption causes information distortion if the non-equidistant distances between adjacent linguistic terms are rigidly transformed to linear equidistant numerical distances. Furthermore, the assessment information given by decision-makers differs from one another and these differences may emanate from the semantics of linguistic terms, distances of adjacent linguistic terms, and the subjective sensations of decision-makers in diverse problems. To date, there have been few 2-tuple linguistic aggregation operators that can correctly reflect these situations.

Motivated by the numerical scales proposed by Dong [20], and taking into account the limitations existing in the 2-tuple linguistic operators mentioned 
above, some new scale functions are proposed in this paper for resolving linguistic translation issues under different semantic and perceptual situations. These scale functions can improve the flexibility and feasibility of modeling linguistic information. Moreover, some 2-tuple linguistic aggregation operators based on these scale functions and a new MCGDM method to avoid the information distortion caused by the transformation from non-equidistant distances of adjacent linguistic terms to linear equidistant numerical distances are proposed; this is in order to reflect the differences of decision-makers' sensations and provide a feasible solution when the linguistic terms are not symmetrically distributed.

The rest of the paper is organized as follows. In Section 2, some basic concepts regarding linguistic 2-tuples are briefly reviewed. In Section 3, the subjective sensation scale based on linguistic term sets, the subjective sensation scale and objective numerical scale based on 2-tuples, and some 2-tuple linguistic aggregation operators based on the subjective sensation scale and objective numerical scale are all proposed. The proposed aggregation operators include the Generated Extended 2-tuple Weighted Averaging (GE2T-WA) operator, Generated Extended 2-Tuple Ordered Weighted Averaging (GE2T-OWA) operator, Generated Extended 2-Tuple Weighted Geometric (GE2T-WG) operator, and Generated Extended 2-Tuple Ordered Weighted Geometric (GE2T-OWG) operator. The MCGDM method based on the GE2TWA and GE2T-OWA operators, or on the GE2T-WG and GE2T-OWA operators, is developed in Section 4. An illustrative example is provided in Section 5.1 and comparisons with other methods are presented in Section 5.2 in order to show validity and feasibility of the proposed approach. Finally, conclusions are drawn in Section 6.

\section{Preliminaries}

In this section, some basic concepts and definitions related to 2-tuples are introduced, including linguistic term sets, the 2-tuple linguistic model and its comparison method, and the canonical characteristic value. These will be utilized in the subsequent analysis.

Let $S=\left\{s_{i} \mid i=0,1, \cdots, 2 g, g \in N^{*}\right\}$ be a linguistic term set, where $N^{*}$ is the set of positive integers and $s_{i}$ represents a possible value for a linguistic variable. The set $S$ satisfies the following properties [16]:

1. The linguistic term set is ordered as: $i>j \Leftrightarrow s_{i}>$ $s_{j}$

2. There is a negation operator which is $\operatorname{Neg}\left(s_{i}\right)=s_{j}$, where $i+j=2 g$. Particularly, $\operatorname{Neg}\left(s_{g}\right)=s_{g}$.

Example 1. A linguistic term set including seven elements can be expressed as follows:

$$
\begin{aligned}
S_{1}=\left\{s_{0}\right. & =\text { Extremely Poor }(\mathrm{EP}), \\
s_{1} & =\operatorname{Very} \text { Poor }(\mathrm{VP}), s_{2}=\operatorname{Poor}(\mathrm{P}), \\
s_{3} & =\operatorname{Medium}(\mathrm{M}), s_{4}=\operatorname{Good}(\mathrm{G}), \\
s_{5} & =\operatorname{Very} \text { Good }(\mathrm{VG}), \\
s_{6} & =\text { Extremely Good }(\mathrm{EG})\} .
\end{aligned}
$$

Herrera and Martinez [16] introduced a 2-tuple linguistic representation model on the basis of symbolic translation, whereby the linguistic information can be represented by linguistic 2-tuples. A 2-tuple can be expressed as $\left(h_{i}, \alpha_{i}\right)$, where $h_{i} \in S$ is a linguistic term and $\alpha_{i} \in[-0.5,0.5)$ is a numerical value showing the deviation between the assessment result and $h_{i}$. The set of 2-tuples is denoted by $\hat{S}$.

Definition 1 [16]. Let $S=\left\{s_{0}, s_{1}, \cdots, s_{2 g}\right\}$ be a linguistic term set, $\beta \in[0,2 g], g$ be a positive integer, and $\beta$ support the result of a symbolic aggregation operation. Then, a 2-tuple $\left(h_{i}, \alpha_{i}\right)\left(h_{i} \in S, \alpha_{i} \in\right.$ $[-0.5,0.5))$, which expresses the equivalent information to $\beta$, can be defined by the following function:

$$
\begin{aligned}
& \Delta:[0,2 g] \rightarrow S \times[-0.5,0.5), \\
& \Delta(\beta)=\left(h_{i}, \alpha_{i}\right),
\end{aligned}
$$

with:

$$
\left\{\begin{array}{l}
h_{i}, i=\operatorname{Round}(\beta) \\
\alpha_{i}=\beta-i
\end{array}\right.
$$

where Round is the usual round operation, $h_{i}$ is the closest index label to $\beta$, and $\alpha_{i}$ is the value of the symbolic translation. $\Delta$ is a function that can convert $\beta$ into $\left(h_{i}, \alpha_{i}\right)$.

Obviously, a linguistic term, $h_{i} \in S$, can be directly converted into a 2-tuple, $\left(h_{i}, 0\right)$.

On the contrary, there is always an inverse function, denoted by $\Delta^{-1}$, so that a linguistic 2-tuple $\left(h_{i}, \alpha_{i}\right)$ can be transformed to its equivalent numerical value $\beta \in[0,2 g] \subseteq R$. The function can be obtained as follows:

$$
\begin{aligned}
& \Delta^{-1}: S \times[-0.5,0.5) \rightarrow[0,2 g] \\
& \Delta^{-1}\left(h_{i}, \alpha_{i}\right)=i+\alpha_{i}=\beta .
\end{aligned}
$$

Definition 2 [16]. Let $\left(h_{l}, \alpha_{m}\right)$ and $\left(h_{k}, \alpha_{n}\right)$ be two 2 -tuples. The comparison methods for two 2-tuples can be described as follows: 
1. If $l<k$, then $\left(h_{l}, \alpha_{m}\right)<\left(h_{k}, \alpha_{n}\right)$;

2. If $l=k$, then:

(a) If $\alpha_{m}<\alpha_{n}$, then $\left(h_{l}, \alpha_{m}\right)<\left(h_{k}, \alpha_{n}\right)$;

(b) If $\alpha_{m}>\alpha_{n}$, then $\left(h_{l}, \alpha_{m}\right)>\left(h_{k}, \alpha_{n}\right)$;

(c) If $\alpha_{m}=\alpha_{n}$, then $\left(h_{l}, \alpha_{m}\right)=\left(h_{k}, \alpha_{n}\right)$.

Definition 3 [32]. Let $S$ be a linguistic term set and $c_{i} \in[0,1](i=0,1, \cdots, 2 g)$ satisfying $c_{0}<c_{1}<\cdots<$ $c_{2 g}$ is called the Canonical Characteristic Value (CCV) of the linguistic term $s_{i} \in S$. Then, the CCV function can be defined as follows:

$$
\begin{aligned}
& \operatorname{CCV}: S \rightarrow[0,1], \\
& \operatorname{CCV}\left(s_{i}\right)=c_{i} .
\end{aligned}
$$

When the linguistic terms are converted to trapezoidal fuzzy numbers $[b-\delta, b, c, c+\delta]$ in the interval $[0,1]$, the $\mathrm{CCV}$ of the linguistic term $s_{i}$ can be obtained as $\operatorname{CCV}\left(s_{i}\right)=(b+c) / 2[32]$.

Example 2. Use the data of Example 1; $s_{i} \in S_{1}$, and the semantics of the linguistic terms can be described by using trapezoidal fuzzy numbers as shown in Table 1.

Therefore, the following results are true:

$$
\begin{aligned}
& \operatorname{CCV}\left(s_{0}\right)=0, \quad \operatorname{CCV}\left(s_{1}\right)=(0.01+0.03) / 2=0.02, \\
& \operatorname{CCV}\left(s_{2}\right)=0.14, \quad \operatorname{CCV}\left(s_{3}\right)=0.5, \\
& \operatorname{CCV}\left(s_{4}\right)=0.86, \quad \operatorname{CCV}\left(s_{5}\right)=0.98, \\
& \operatorname{CCV}\left(s_{6}\right)=1 .
\end{aligned}
$$

3. Some new linguistic scales and 2-tuple linguistic aggregation operators based on the subjective sensation scale and objective numerical scale

The assessment information given by decision-makers is subjective, which is also significantly distinctive

Table 1. The conversion of linguistic terms into trapezoidal fuzzy numbers.

\begin{tabular}{cl}
\hline Linguistic terms & Trapezoidal fuzzy numbers \\
\hline EP & {$[0,0,0,0]$} \\
VP & {$[0,0.01,0.03,0.04]$} \\
P & {$[0.03,0.10,0.18,0.25]$} \\
M & {$[0.36,0.42,0.58,0.64]$} \\
G & {$[0.75,0.82,0.90,0.97]$} \\
VG & {$[0.96,0.97,0.99,1]$} \\
EG & {$[1,1,1,1]$} \\
\hline
\end{tabular}

between different decision-makers regarding the semantics of linguistic terms and distances of adjacent linguistic terms, and also depends on the subjective sensations in different decision-making problems; therefore, the linguistic terms may not be symmetrically distributed and the distances between adjacent linguistic terms are often unequal. For solving these problems, the subjective sensation scale based on linguistic term sets, the subjective sensation scale and objective numerical scale based on 2-tuples, and some 2-tuple linguistic aggregation operators based on the subjective sensation scale and objective numerical scale are proposed in this section.

\subsection{The subjective sensation scale based on linguistic term sets}

In this subsection, the subjective sensation scale based on linguistic term sets is improved. The scale can reflect the differences between the semantics of linguistic terms, distances of adjacent linguistic terms, and the differences between the decision-makers' sensations in diverse situations.

In order to preserve all the information provided in the decision-making process and facilitate the calculation, the linguistic term set $S=\left\{s_{i} \mid i=\right.$ $\left.0,1, \cdots, 2 g, g \in N^{*}\right\}$ is replaced with the extended linguistic term set $\tilde{S}=\left\{s_{i} \mid i=0,1, \cdots, t, t \in N^{*}\right\}$, where $s_{i}>s_{j}(i>j), t \geq 2 g$, and $N^{*}$ is the set of positive integers. Correspondingly, the extended set of 2-tuples is denoted by $\bar{S}=\left\{\left(h_{i}, \alpha_{i}\right) \mid i=0,1, \cdots, t, t \in\right.$ $\left.N^{*}\right\}$, where $h_{i} \in \tilde{S}$, and $\alpha_{i} \in[-0.5,0.5)$. If the linguistic term is $s_{i} \in S$, then it is referred to as the original linguistic term; otherwise, $s_{i}$ is called the virtual linguistic term. In general, decision-makers use original linguistic terms to evaluate alternatives with virtual linguistic terms only appearing in the operations to avoid information loss and enhance the decision-making process [51].

Definition 4. Let $S=\left\{s_{i} \mid i=0,1, \cdots, 2 g, g \in N^{*}\right\}$ be a linguistic term set, $\tilde{S}=\left\{s_{i} \mid i=0,1, \cdots, t, t \in N^{*}\right\}$ be an extended linguistic term set, and $R$ be the set of real numbers. Then, the subjective sensation function of $\tilde{S}:$ SNS : $\tilde{S} \rightarrow R$ can be defined as follows:

$$
\operatorname{SNS}\left(s_{i}\right)= \begin{cases}-g, & i=0 \\ -g+\frac{2 g \times \sum_{j=1}^{i} \operatorname{dist}\left(s_{j-1}, s_{j}\right)}{\sum_{j=1}^{2 g} \operatorname{dist}\left(s_{j-1}, s_{j}\right)}, & i=1,2, \cdots, t\end{cases}
$$

where $\operatorname{dist}\left(s_{j-1}, s_{j}\right) \quad(j=1,2, \cdots, t)$ is the subjective distances of the adjacent linguistic terms $s_{j-1}$ and $s_{j}$. $\operatorname{dist}\left(s_{j-1}, s_{j}\right)$ can be directly provided by the decision-makers, and it can also be obtained by the semantics of linguistic terms, e.g. $\operatorname{dist}\left(s_{j-1}, s_{j}\right)=$ $\operatorname{CCV}\left(s_{j}\right)-\operatorname{CCV}\left(s_{j-1}\right)$ [32]. If $j>2 g$, then $\operatorname{CCV}\left(s_{j}\right)=$ 
$\eta \operatorname{CCV}\left(s_{2 g}\right)+\operatorname{CCV}\left(s_{j-2 \eta g}\right)$, where $\eta=\operatorname{int}(j / 2 g)$ and int(.) is the bracket function.

Example 3. Use the data of Example 2 and let the extended linguistic term set be $\tilde{S}_{1}=\left\{s_{i} \mid i=\right.$ $0,1, \cdots, 8\}$.

Therefore, the CCVs of $s_{i}(i=0,1, \cdots, 8)$ can be calculated as follows:

$$
\begin{aligned}
& \operatorname{CCV}\left(s_{0}\right)=0, \\
& \operatorname{CCV}\left(s_{1}\right)=(0.01+0.03) / 2=0.02, \\
& \operatorname{CCV}\left(s_{2}\right)=0.14, \quad \operatorname{CCV}\left(s_{3}\right)=0.5, \\
& \operatorname{CCV}\left(s_{4}\right)=0.86, \quad \operatorname{CCV}\left(s_{5}\right)=0.98, \\
& \operatorname{CCV}\left(s_{6}\right)=1, \\
& \operatorname{CCV}\left(s_{7}\right)=\operatorname{CCV}\left(s_{6}\right)+\operatorname{CCV}\left(s_{1}\right)=1.02, \\
& \operatorname{CCV}\left(s_{8}\right)=\operatorname{CCV}\left(s_{6}\right)+\operatorname{CCV}\left(s_{2}\right)=1.14 .
\end{aligned}
$$

The subjective distances of the adjacent linguistic terms, $\operatorname{dist}\left(s_{j-1}, s_{j}\right)(j=1,2, \cdots, 8)$, can be obtained according to the CCVs of $s_{i}(i=0,1, \cdots, 8)$ as:

$$
\begin{aligned}
& \operatorname{dist}\left(s_{0}, s_{1}\right)=\operatorname{CCV}\left(s_{1}\right)-\operatorname{CCV}\left(s_{0}\right)=0.02, \\
& \operatorname{dist}\left(s_{1}, s_{2}\right)=0.12, \quad \operatorname{dist}\left(s_{2}, s_{3}\right)=0.36, \\
& \operatorname{dist}\left(s_{3}, s_{4}\right)=0.36, \quad \operatorname{dist}\left(s_{4}, s_{5}\right)=0.12, \\
& \operatorname{dist}\left(s_{5}, s_{6}\right)=0.02, \quad \operatorname{dist}\left(s_{6}, s_{7}\right)=0.02, \\
& \operatorname{dist}\left(s_{7}, s_{8}\right)=0.12 .
\end{aligned}
$$

Therefore, the subjective sensation scales of $s_{i}(i=$ $0,1, \cdots, 8)$ can be obtained as follows:

$$
\begin{aligned}
\operatorname{SNS}\left(s_{0}\right) & =-3, \\
\operatorname{SNS}\left(s_{1}\right) & =-3+\frac{6 \times \sum_{j=1}^{1} \operatorname{dist}\left(s_{j-1}, s_{j}\right)}{\sum_{j=1}^{6} \operatorname{dist}\left(s_{j-1}, s_{j}\right)} \\
& =-3+\frac{6 \times 0.02}{1}=-2.88, \\
\operatorname{SNS}\left(s_{2}\right) & =-2.16, \quad \operatorname{SNS}\left(s_{3}\right)=0, \\
\operatorname{SNS}\left(s_{4}\right) & =2.16, \quad \operatorname{SNS}\left(s_{5}\right)=2.88, \\
\operatorname{SNS}\left(s_{6}\right) & =3, \\
\operatorname{SNS}\left(s_{8}\right) & =3.84 .
\end{aligned}
$$

Theorem 1. If $s_{1}<s_{k}$ for $\forall s_{l}, s_{k} \in \tilde{S}$, then $\operatorname{SNS}\left(s_{l}\right)<\operatorname{SNS}\left(s_{k}\right)$.

Proof. If $s_{l}<s_{k}$ for $\forall s_{l}, s_{k}$, then two cases need to be discussed.

1. If $0=l<k$, then:

$$
\begin{gathered}
\operatorname{SNS}\left(s_{k}\right)-\operatorname{SNS}\left(s_{l}\right)=-g+\frac{2 g \times \sum_{j=1}^{k} \operatorname{dist}\left(s_{j-1}, s_{j}\right)}{\sum_{j=1}^{2 g} \operatorname{dist}\left(s_{j-1}, s_{j}\right)} \\
-(-g)=\frac{2 g \times \sum_{j=1}^{k} \operatorname{dist}\left(s_{j-1}, s_{j}\right)}{\sum_{j=1}^{2 g} \operatorname{dist}\left(s_{j-1}, s_{j}\right)}>0 .
\end{gathered}
$$

2. If $0<l<k$, then:

$$
\begin{aligned}
\operatorname{SNS}\left(s_{k}\right)-\operatorname{SNS}\left(s_{l}\right)=-g \\
+\frac{2 g \times \sum_{j=1}^{k} \operatorname{dist}\left(s_{j-1}, s_{j}\right)}{\sum_{j=1}^{2 g} \operatorname{dist}\left(s_{j-1}, s_{j}\right)} \\
-\left(\begin{array}{l}
\left.-g+\frac{2 g \times \sum_{j=1}^{l} \operatorname{dist}\left(s_{j-1}, s_{j}\right)}{\sum_{j=1}^{2 g} \operatorname{dist}\left(s_{j-1}, s_{j}\right)}\right) \\
= \\
2 g \times \sum_{j=l}^{k} \operatorname{dist}\left(s_{j-1}, s_{j}\right) \\
\sum_{j=1}^{2 g} \operatorname{dist}\left(s_{j-1}, s_{j}\right)
\end{array} 0 .\right.
\end{aligned}
$$

Thus, $\operatorname{SNS}\left(s_{l}\right)<\operatorname{SNS}\left(s_{k}\right)$.

\subsection{The subjective sensation scale based on 2-tuples}

In this subsection, the subjective sensation scale based on 2-tuples is proposed. It can solve problems regarding differences between the semantics of linguistic terms and distances of adjacent linguistic terms, and also differences between the decision-makers' sensations in diverse situations. Moreover, it is feasible for situations regarding assessment information in the form of 2 tuples.

Definition 5. Let $\bar{S}$ be a set of 2-tuples and $R$ be the set of real numbers. Then, the subjective sensation function of $\bar{S}$ can be defined as follows: 
$\overline{\mathrm{SNS}}: \bar{S} \rightarrow R$,

$\overline{\operatorname{SNS}}((h, \alpha))=\left\{\begin{array}{c}\operatorname{SNS}\left(s_{j}\right)+\alpha \times\left(\operatorname{SNS}\left(s_{j+1}\right)-\operatorname{SNS}\left(s_{j}\right)\right), \\ \alpha \geq 0 \text { and } h=s_{j} \\ \operatorname{SNS}\left(s_{j}\right)+\alpha \times\left(\operatorname{SNS}\left(s_{j}\right)-\operatorname{SNS}\left(s_{j-1}\right)\right), \\ \alpha<0 \text { and } h=s_{j}\end{array}\right.$

where $s_{j}, h \in \tilde{S}$ and $(h, \alpha) \in \bar{S}$.

Example 4. Use the data of Example 1, Let $\bar{S}_{1}$ be an extended 2-tuple linguistic set, $\left(s_{0}, 0.1\right),\left(s_{1},-0.2\right) \in$ $\bar{S}_{1}\left(s_{0}, s_{1} \in S_{1}\right)$, and the subjective distances of the adjacent linguistic terms be $\operatorname{dist}\left(s_{j-1}, s_{j}\right)=1$.

$\overline{\operatorname{SNS}}\left(\left(s_{0}, 0.1\right)\right)=\operatorname{SNS}\left(s_{0}\right)+0.1 \times\left(\operatorname{SNS}\left(s_{1}\right)-\operatorname{SNS}\left(s_{0}\right)\right)$

$$
=-3+0.1 \times(-2-(-3))=-2.9,
$$

$\overline{\operatorname{SNS}}\left(\left(s_{1},-0.2\right)\right)=\operatorname{SNS}\left(s_{1}\right)-0.2 \times\left(\operatorname{SNS}\left(s_{1}\right)-\operatorname{SNS}\left(s_{0}\right)\right)$

$$
=-2-0.2 \times(-2-(-3))=-2.2 .
$$

Theorem 2. If $\left(h_{l}, \alpha_{m}\right)<\left(h_{k}, \alpha_{n}\right)$ for $\forall\left(h_{l}, \alpha_{m}\right)$, $\left(h_{k}, \alpha_{n}\right) \in \bar{S}$, then $\overline{\operatorname{SNS}}\left(\left(h_{l}, \alpha_{m}\right)\right)<\overline{\operatorname{SNS}}\left(\left(h_{k}, \alpha_{n}\right)\right)$.

Proof. For $\forall\left(h_{l}, \alpha_{m}\right),\left(h_{k}, \alpha_{n}\right)$, if $\left(h_{l}, \alpha_{m}\right)<\left(h_{k}, \alpha_{n}\right)$, then $h_{l}<h_{k}$, or $h_{l}=h_{k}$ and $\alpha_{m}<\alpha_{n}$.

1. If $h_{l}<h_{k}$, then $k-1 \geq l$, i.e. $k \geq l+1$. According to Theorem 1, $\operatorname{SNS}\left(h_{k-1}\right) \geq \operatorname{SNS}\left(h_{l}\right)$ and $\operatorname{SNS}\left(h_{k}\right) \geq \operatorname{SNS}\left(h_{l+1}\right)$ can be obtained.

$$
\begin{aligned}
\overline{\mathrm{SNS}} & \left(\left(h_{k}, \alpha_{n}\right)\right)-\overline{\mathrm{SNS}}\left(\left(h_{l}, \alpha_{m}\right)\right) \geq \operatorname{SNS}\left(h_{k}\right)+\alpha_{n} \\
& \times\left(\operatorname{SNS}\left(h_{k}\right)-\operatorname{SNS}\left(h_{k-1}\right)\right) \\
& -\left(\operatorname{SNS}\left(h_{l}\right)+\alpha_{m} \times\left(\operatorname{SNS}\left(h_{l+1}\right)-\operatorname{SNS}\left(h_{l}\right)\right)\right) \\
& >\operatorname{SNS}\left(h_{k}\right)-\operatorname{SNS}\left(h_{l}\right)-0.5 \\
& \times\left(\operatorname{SNS}\left(h_{k}\right)-\operatorname{SNS}\left(h_{k-1}\right)\right) \\
& -0.5 \times\left(\operatorname{SNS}\left(h_{l+1}\right)-\operatorname{SNS}\left(h_{l}\right)\right) \\
& >\operatorname{SNS}\left(h_{k}\right)-\operatorname{SNS}\left(h_{l}\right)-0.5 \\
& \times\left(\operatorname{SNS}\left(h_{k}\right)-\operatorname{SNS}\left(h_{k-1}\right)\right) \\
& -0.5 \times\left(\operatorname{SNS}\left(h_{k}\right)-\operatorname{SNS}\left(h_{l}\right)\right) \\
& =0.5 \times\left(\operatorname{SNS}\left(h_{k}\right)-\operatorname{SNS}\left(h_{l}\right)\right)-0.5 \\
& \times\left(\operatorname{SNS}\left(h_{k}\right)-\operatorname{SNS}\left(h_{k-1}\right)\right) \\
& =0.5 \times\left(\operatorname{SNS}\left(h_{k-1}\right)-\operatorname{SNS}\left(h_{l}\right)\right) \geq 0 .
\end{aligned}
$$

2. If $h_{l}=h_{k}$, then three cases need to be discussed:

(a) If $0<\alpha_{m}<\alpha_{n}$, then:

$$
\begin{aligned}
& \overline{\mathrm{SNS}}\left(\left(h_{k}, \alpha_{n}\right)\right)-\overline{\operatorname{SNS}}\left(\left(h_{l}, \alpha_{m}\right)\right)=\operatorname{SNS}\left(h_{k}\right) \\
& +\alpha_{n} \times\left(\operatorname{SNS}\left(h_{k+1}\right)-\operatorname{SNS}\left(h_{k}\right)\right) \\
& -\left(\operatorname{SNS}\left(h_{l}\right)+\alpha_{m} \times\left(\operatorname{SNS}\left(h_{l+1}\right)\right.\right. \\
& \left.\left.-\operatorname{SNS}\left(h_{l}\right)\right)\right)=\left(\alpha_{n}-\alpha_{m}\right)\left(\operatorname{SNS}\left(h_{l+1}\right)\right. \\
& \left.-\operatorname{SNS}\left(h_{l}\right)\right)>0 .
\end{aligned}
$$

(b) If $\alpha_{m}<0<\alpha_{n}$, then:

$$
\begin{aligned}
\overline{\mathrm{SNS}}\left(\left(h_{k}, \alpha_{n}\right)\right)-\overline{\operatorname{SNS}}\left(\left(h_{l}, \alpha_{m}\right)\right) \\
=\operatorname{SNS}\left(h_{k}\right)+\alpha_{n} \times\left(\operatorname{SNS}\left(h_{k+1}\right)\right. \\
\left.\quad-\operatorname{SNS}\left(h_{k}\right)\right)-\left(\operatorname{SNS}\left(h_{l}\right)+\alpha_{m}\right. \\
\left.\quad \times\left(\operatorname{SNS}\left(h_{l}\right)-\operatorname{SNS}\left(h_{l-1}\right)\right)\right)>0 \\
\quad \times\left(\operatorname{SNS}\left(h_{k+1}\right)-\operatorname{SNS}\left(h_{k}\right)\right)-\alpha_{m} \\
\quad \times\left(\operatorname{SNS}\left(h_{l}\right)-\operatorname{SNS}\left(h_{l-1}\right)\right)>0 .
\end{aligned}
$$

(c) If $\alpha_{m}<\alpha_{n}<0$, then:

$$
\begin{aligned}
& \overline{\operatorname{SNS}}\left(\left(h_{k}, \alpha_{n}\right)\right)-\overline{\operatorname{SNS}}\left(\left(h_{l}, \alpha_{m}\right)\right)=\operatorname{SNS}\left(h_{k}\right) \\
& +\alpha_{n} \times\left(\operatorname{SNS}\left(h_{k}\right)-\operatorname{SNS}\left(h_{k-1}\right)\right) \\
& -\left(\operatorname{SNS}\left(h_{l}\right)+\alpha_{m} \times\left(\operatorname{SNS}\left(h_{l}\right)\right.\right. \\
& \left.\left.\quad-\operatorname{SNS}\left(h_{l-1}\right)\right)\right)=\left(\alpha_{n}-\alpha_{m}\right)\left(\operatorname{SNS}\left(h_{k}\right)\right. \\
& \left.-\operatorname{SNS}\left(h_{k-1}\right)\right)>0 .
\end{aligned}
$$

Therefore, $\overline{\operatorname{SNS}}\left(\left(h_{l}, \alpha_{m}\right)\right)<\overline{\operatorname{SNS}}\left(\left(h_{k}, \alpha_{n}\right)\right)$.

Definition 6. Let $Q$ be the set of $\overline{\operatorname{SNS}}\left(\left(h_{i}, \alpha_{i}\right)\right)$. Then, the approximation function can be defined as follows:

$$
\begin{aligned}
& \Phi: Q \rightarrow \tilde{S}, \\
& \Phi\left(\theta_{k}\right)=\max _{i}\left\{h_{i}\right\},
\end{aligned}
$$

where $h_{i}$ satisfies $\left|\operatorname{SNS}\left(h_{i}\right)-\theta_{k}\right|=\min _{j}\left\{\left|\theta_{k}-\operatorname{SNS}\left(h_{j}\right)\right|\right\}$, $h_{i}, h_{j} \in \tilde{S}$, and $\theta_{k} \in Q$.

Example 5. Use the data of Example 1, and let the distance of the adjacent linguistic terms be 
$\operatorname{dist}\left(s_{j-1}, s_{j}\right)=1$ and $\theta_{1}=1.5$. According to Definition 6:

$$
\min _{j}\left\{\left|1.5-\operatorname{SNS}\left(s_{j}\right)\right|\right\}=0.5
$$

can be obtained.

Since $\operatorname{SNS}\left(s_{4}\right)=1$ and $\operatorname{SNS}\left(s_{5}\right)=2, \mid \operatorname{SNS}\left(s_{4}\right)-$ $1.5|=| \operatorname{SNS}\left(s_{5}\right)-1.5 \mid=0.5$ can be obtained.

Therefore, we have $\Phi\left(\theta_{1}\right)=\max _{i}\left\{s_{4}, s_{5}\right\}=s_{5}$.

Theorem 3. Let $Q$ be the set of $\overline{\operatorname{SNS}}\left(\left(h_{i}, \alpha_{i}\right)\right)$ and SNS be the subjective sensation function of $\tilde{S}$. There is always an inverse function of $\overline{\mathrm{SNS}}$ denoted by $\overline{\mathrm{SNS}}^{-1}$.

$$
\overline{\mathrm{SNS}}^{-1}\left(\theta_{k}\right)=\left(h_{i}, \alpha_{i}\right),
$$

where:

$$
\begin{aligned}
& h_{i}= \Phi\left(\theta_{k}\right), \\
& \alpha_{i}= \begin{cases}\frac{\theta_{k}-\operatorname{SNS}\left(s_{j}\right)}{\operatorname{SNS}\left(s_{j+1}\right)-\operatorname{SNS}\left(s_{j}\right)}, & \theta_{k} \geq \operatorname{SNS}\left(s_{j}\right) \quad \text { and } \\
\frac{\theta_{k}-\operatorname{SNS}\left(s_{j}\right)}{\operatorname{SNS}\left(s_{j}\right)-\operatorname{SNS}\left(s_{j-1}\right)}, & \theta_{j}<h_{i} \\
& s_{j}=h_{i}\end{cases}
\end{aligned}
$$

Proof. According to Theorem 3 and Definition 5, the fact that the function is a strictly monotonically increasing and continuous function can be obtained.

1. Since $\theta_{k}=\overline{\mathrm{SNS}}\left(\left(h_{i}, \alpha_{i}\right)\right), \overline{\mathrm{SNS}}^{-1}\left(\theta_{k}\right)=\overline{\mathrm{SNS}}^{-1}$ $\left(\overline{\mathrm{SNS}}\left(\left(h_{i}, \alpha_{i}\right)\right)\right)=\left(h_{i}, \alpha_{i}\right)$ can be obtained;

2. Since $\left(h_{i}, \alpha_{i}\right)=\overline{\operatorname{SNS}}^{-1}\left(\theta_{k}\right), \overline{\operatorname{SNS}}\left(\left(h_{i}, \alpha_{i}\right)\right)=$ $\overline{\mathrm{SNS}}\left(\overline{\mathrm{SNS}}^{-1}\left(\theta_{k}\right)\right)=\theta_{k}$ can be obtained.

Therefore, there is always an inverse function $\overline{\mathrm{SNS}}^{-1}\left(\theta_{k}\right)=\left(h_{i}, \alpha_{i}\right)$.

Example 6. Use the data of Example $5, \Phi\left(\theta_{1}\right)=$ $\max _{i}\left\{s_{4}, s_{5}\right\}=s_{5}$.

Since $1.5<\operatorname{SNS}\left(s_{5}\right)=2$, we have:

$$
\alpha_{5}=\frac{1.5-\operatorname{SNS}\left(s_{5}\right)}{\operatorname{SNS}\left(s_{5}\right)-\operatorname{SNS}\left(s_{4}\right)}=\frac{1.5-2}{2-1}=-0.5 \text {. }
$$

Therefore, $\overline{\mathrm{SNS}}^{-1}(1.5)=\left(s_{5},-0.5\right)$ can be obtained.

Theorem 4. If $\theta_{l}<\theta_{k}$ for $\forall \theta_{l}, \theta_{k} \in Q$, then $\overline{\mathrm{SNS}}^{-1}$ $\left(\theta_{l}\right)<\overline{\mathrm{SNS}}^{-1}\left(\theta_{k}\right)$.

Proof. If $\forall \theta_{l}, \theta_{k}, \theta_{l}<\theta_{k}, \overline{\operatorname{SNS}}\left(\left(h_{l}, \alpha_{m}\right)\right)<\overline{\mathrm{SNS}}\left(\left(h_{k}\right.\right.$, $\left.\left.\alpha_{n}\right)\right)$ can be obtained. According to the conclusion, the inequality of Theorem 4 can be proved as follows:
1. If $h_{l}=\Phi\left(\theta_{l}\right)=\Phi\left(\theta_{k}\right)=h_{k}$, then $\alpha_{m}<\alpha_{n}$ and $\overline{\mathrm{SNS}}^{-1}\left(\theta_{l}\right)=\left(h_{l}, \alpha_{m}\right)<\left(h_{k}, \alpha_{n}\right)=\overline{\mathrm{SNS}}^{-1}\left(\theta_{k}\right)$;

2. If $h_{l}=\Phi\left(\theta_{l}\right)<\Phi\left(\theta_{k}\right)=h_{k}$, then $\overline{\mathrm{SNS}}^{-1}\left(\theta_{l}\right)=$ $\left(h_{l}, \alpha_{m}\right)<\left(h_{k}, \alpha_{n}\right)=\overline{\mathrm{SNS}}^{-1}\left(\theta_{k}\right)$.

Therefore, $\overline{\mathrm{SNS}}^{-1}\left(\theta_{l}\right)<\overline{\mathrm{SNS}}^{-1}\left(\theta_{k}\right)$.

\subsection{The objective numerical scale based on 2-tuples}

In this subsection, the objective numerical scale based on 2-tuples is introduced in order to normalize the data that is in the form of a subjective sensation scale, which can process the data more accurately.

Definition 7. The objective numerical function of $\bar{S}$ can be defined as follows:

$$
\begin{aligned}
& \Gamma: \bar{S} \rightarrow[0,1], \\
& \Gamma((h, \alpha))=\left\{\begin{array}{c}
\frac{a^{-\operatorname{SNS}\left(s_{0}\right)}+a^{\overline{\operatorname{SNS}}((h, \alpha))}-2}{2 a^{\operatorname{SNS}\left(s_{2}\right)}-2} \overline{\operatorname{SNS}}((h, \alpha)) \geq 0 \\
\frac{-a^{-\overline{\operatorname{SNS}}((h, \alpha))}+a^{-\operatorname{SNS}\left(s_{0}\right)}}{2 a^{\operatorname{SNS}\left(s_{2}\right)}-2} \overline{\operatorname{SNS}}((h, \alpha))<0
\end{array}\right.
\end{aligned}
$$

where $a>1, s_{0}, s_{2 g}, h \in \tilde{S}$ and $(h, \alpha) \in \bar{S}$.

Different scales can be obtained by setting $a$. There are two methods, namely the experimental method and subjective method, for determining the value of $a$ and usually $a \in[1.36,1.4][52,53]$.

Example 7. Use the data of Example 1, let $\bar{S}_{2}$ be an extended 2-tuple linguistic term set, the subjective distances of the adjacent evaluation values be $\operatorname{dist}\left(s_{j-1}, s_{j}\right)=1,\left(s_{0}, 0.2\right),\left(s_{3}, 0\right) \in \bar{S}_{2}$, and $a=1.4$.

Since $\overline{\operatorname{SNS}}\left(\left(s_{0}, 0.2\right)\right)=-2.8<0$,

Then:

$$
\begin{aligned}
\Gamma\left(\left(s_{0}, 0.2\right)\right) & =\frac{-1.4^{-\overline{\mathrm{SNS}}\left(\left(s_{0}, 0.2\right)\right)}+1.4^{-\mathrm{SNS}\left(s_{0}\right)}}{2 \times 1.4^{\mathrm{SNS}\left(s_{6}\right)}-2} \\
& =\frac{-1.4^{2.8}+1.4^{3}}{2 \times 1.4^{3}-2}=0.052
\end{aligned}
$$

can be obtained. Similarly:

$$
\overline{\operatorname{SNS}}\left(\left(s_{3}, 0\right)\right)=0 \geq 0,
$$

and thus:

$$
\begin{aligned}
\Gamma\left(\left(s_{3}, 0\right)\right) & =\frac{1.4^{-\mathrm{SNS}\left(s_{0}\right)}+1.4^{\overline{\mathrm{SNS}}\left(\left(s_{3}, 0\right)\right)}-2}{2 \times 1.4^{\mathrm{SNS}\left(s_{6}\right)}-2} \\
& =\frac{1.4^{3}+1.4^{0}-2}{2 \times 1.4^{3}-2}=0.5 .
\end{aligned}
$$


Theorem 5. For $\forall\left(h_{l}, \alpha_{m}\right),\left(h_{k}, \alpha_{n}\right) \in \bar{S}$, if $\left(h_{l}, \alpha_{m}\right)$ $<\left(h_{k}, \alpha_{n}\right), \Gamma\left(\left(h_{l}, \alpha_{m}\right)\right)<\Gamma\left(\left(h_{k}, \alpha_{n}\right)\right)$.

Proof. For $\forall\left(h_{l}, \alpha_{m}\right),\left(h_{k}, \alpha_{n}\right)$, if $\left(h_{l}, \alpha_{m}\right)<\left(h_{k}, \alpha_{n}\right)$ and $a>1, \overline{\operatorname{SNS}}\left(\left(h_{l}, \alpha_{m}\right)\right)<\overline{\operatorname{SNS}}\left(\left(h_{k}, \alpha_{n}\right)\right)$ and $a^{\overline{\mathrm{SNS}}\left(\left(h_{k}, \alpha_{n}\right)\right)}+a^{\overline{\mathrm{SNS}}\left(\left(h_{l}, \alpha_{m}\right)\right)}-2>0$ can be obtained. According to the conclusion, the inequality of Theorem 5 can be proved as follows:

1. If $0<\overline{\operatorname{SNS}}\left(\left(h_{l}, \alpha_{m}\right)\right)<\overline{\operatorname{SNS}}\left(\left(h_{k}, \alpha_{n}\right)\right)$, then:

$$
\begin{aligned}
\overline{\mathrm{SNS}}\left(\left(h_{k}, \alpha_{n}\right)\right)-\overline{\mathrm{SNS}}\left(\left(h_{l}, \alpha_{m}\right)\right) \\
=\frac{a^{-\mathrm{SNS}\left(s_{0}\right)}+a^{\overline{\mathrm{SNS}}\left(\left(h_{k}, \alpha_{n}\right)\right)}-2}{2 a^{\mathrm{SNS}\left(s_{2 g}\right)}-2} \\
-\frac{a^{-\mathrm{SNS}\left(s_{0}\right)}+a^{\overline{\mathrm{SNS}}\left(\left(h_{l}, \alpha_{m}\right)\right)}-2}{2 a^{\mathrm{SNS}\left(s_{2 g}\right)}-2} \\
=\frac{a^{\overline{\mathrm{SNS}}\left(\left(h_{k}, \alpha_{n}\right)\right)}-a^{\overline{\mathrm{SNS}}\left(\left(h_{l}, \alpha_{m}\right)\right)}}{2 a^{\mathrm{SNS}\left(s_{2}\right)}-2}>0 .
\end{aligned}
$$

2. If $\overline{\operatorname{SNS}}\left(\left(h_{l}, \alpha_{m}\right)\right)<0<\left(\left(h_{k}, \alpha_{n}\right)\right)$, then:

$$
\begin{aligned}
\overline{\mathrm{SNS}}\left(\left(h_{k}, \alpha_{n}\right)\right)-\overline{\mathrm{SNS}}\left(\left(h_{l}, \alpha_{m}\right)\right) \\
=\frac{a^{-\mathrm{SNS}\left(s_{0}\right)}+a^{\overline{\mathrm{SNS}}\left(\left(h_{k}, \alpha_{n}\right)\right)}-2}{2 a^{\mathrm{SNS}\left(s_{2 g}\right)}-2} \\
-\frac{-a^{-\overline{\mathrm{SNS}}\left(\left(h_{l}, \alpha_{m}\right)\right)}+a^{-\mathrm{SNS}\left(s_{0}\right)}}{2 a^{\mathrm{SNS}\left(s_{2 g}\right)}-2} \\
=\frac{a^{\overline{\mathrm{SNS}}\left(\left(h_{k}, \alpha_{n}\right)\right)}+a^{-\overline{\mathrm{SNS}}\left(\left(h_{l}, \alpha_{m}\right)\right)}-2}{2 a^{\mathrm{SNS}\left(s_{2 g}\right)}-2}>0 .
\end{aligned}
$$

3. If $\overline{\operatorname{SNS}}\left(\left(h_{l}, \alpha_{m}\right)\right)<\overline{\operatorname{SNS}}\left(\left(h_{k}, \alpha_{n}\right)\right)<0$, then:

$$
\begin{aligned}
& \overline{\mathrm{SNS}}\left(\left(h_{k}, \alpha_{n}\right)\right)-\overline{\mathrm{SNS}}\left(\left(h_{l}, \alpha_{m}\right)\right) \\
& =\frac{-a^{-\overline{\mathrm{SNS}}\left(\left(h_{k}, \alpha_{n}\right)\right)}+a^{-\mathrm{SNS}\left(s_{0}\right)}}{2 a^{\mathrm{SNS}\left(s_{2 g}\right)}-2} \\
& -\frac{-a^{-\overline{\mathrm{SNS}}\left(\left(h_{l}, \alpha_{m}\right)\right)}+a^{-\mathrm{SNS}\left(s_{0}\right)}}{2 a^{\mathrm{SNS}\left(s_{2 g}\right)}-2} \\
& =\frac{-a^{-\overline{\mathrm{SNS}}\left(\left(h_{k}, \alpha_{n}\right)\right)}+a^{-\overline{\mathrm{SNS}}\left(\left(h_{l}, \alpha_{m}\right)\right)}}{2 a^{\mathrm{SNS}\left(s_{2 g}\right)}-2}>0 .
\end{aligned}
$$

Therefore, $\Gamma\left(\left(h_{l}, \alpha_{m}\right)\right)<\Gamma\left(\left(h_{k}, \alpha_{n}\right)\right)$.

Theorem 6. Let $U$ be the set of $\Gamma((h, \alpha)),(h, \alpha) \in \bar{S}$, and $\gamma \in U$. Then, there is always an inverse function of $\Gamma$ denoted by $\Gamma^{-1}$.

$$
\Gamma^{-1}(\gamma)=\left\{\begin{aligned}
& \overline{\mathrm{SNS}}^{-1}\left(\operatorname { l o g } _ { a } \left(\gamma\left(2 a^{\mathrm{SNS}\left(s_{2 g}\right)}-2\right)\right.\right. \\
&\left.\left.-a^{-\mathrm{SNS}\left(s_{0}\right)}+2\right)\right) \\
& \gamma \geq 0.5 \\
& \overline{\mathrm{SNS}}^{-1}\left(-\log _{a}\left(-\gamma\left(2 a^{\mathrm{SNS}\left(s_{2 g}\right)}-2\right)\right.\right. \\
& \\
&\left.\left.+a^{-\mathrm{SNS}\left(s_{0}\right)}\right)\right) \\
& \gamma<0.5
\end{aligned}\right.
$$

where $a>1$.

For proof of $\Gamma^{-1}$, one can refer to Theorem 3 .

Example 8. Use the data of Example 1, let the subjective distances of the adjacent evaluation values be $\operatorname{dist}\left(s_{j-1}, s_{j}\right)=1, a=1.4, \gamma_{1}, \gamma_{2} \in U, \gamma_{1}=0.455$, and $\gamma_{2}=0.587$.

Because $\gamma=0.455<0.5$, the following can be obtained:

$$
\begin{aligned}
\Gamma^{-1}(0.455) \\
\quad=\overline{\operatorname{SNS}}^{-1}\left(-\log _{1.4}\left(-0.455 \times\left(2 \times 1.4^{3}-2\right)+1.4^{3}\right)\right) \\
\quad=\overline{\operatorname{SNS}}^{-1}(-0.431)=\left(s_{3},-0.431\right) .
\end{aligned}
$$

Similarly, $\gamma=0.587 \geq 0.5$, and thus:

$$
\begin{aligned}
\Gamma^{-1} & (0.587) \\
& =\overline{\operatorname{SNS}}^{-1}\left(\log _{1.4}\left(0.587\left(2 \times 1.4^{3}-2\right)-1.4^{3}+2\right)\right) \\
& =\overline{\operatorname{SNS}}^{-1}(0.788)=\left(s_{4},-0.212\right) .
\end{aligned}
$$

Theorem 7. For $\forall \gamma_{l}, \gamma_{k} \in U$, if $\gamma_{l}<\gamma_{k}$, then $\Gamma^{-1}\left(\gamma_{l}\right)<\Gamma^{-1}\left(\gamma_{k}\right)$.

For proof of $\Gamma^{-1}\left(\gamma_{l}\right)<\Gamma^{-1}\left(\gamma_{k}\right)$ in the case of $\gamma_{l}<\gamma_{k}$, one can refer to Theorem 4 .

\subsection{2-tuple linguistic aggregation operators based on the subjective sensation and objective numerical scales}

Some operators are proposed in this subsection that are based on the functions $\Gamma$ and $\Gamma^{-1}$.

Definition 8. Let $\left(h_{i}, \alpha_{i}\right)(i=1,2 \cdots, n)$ be a set of $n$ 2-tuples, which has an associated weight vector $w=$ $\left(\left(w_{1}, \beta_{1}\right),\left(w_{2}, \beta_{2}\right), \cdots,\left(w_{n}, \beta_{n}\right)\right)$. Then, the GE2TWA operator can be defined as follows:

$$
\begin{aligned}
& \operatorname{GE2T-WA}: \bar{S}^{n} \rightarrow \bar{S}, \\
& \operatorname{GE2T}-\operatorname{WA}\left(\left(h_{1}, \alpha_{1}\right),\left(h_{2}, \alpha_{2}\right), \cdots,\left(h_{n}, \alpha_{n}\right)\right) \\
&=\Gamma^{-1}\left(\left(\sum_{i=1}^{n} \frac{\Gamma\left(\left(w_{i}, \beta_{i}\right)\right) \Gamma^{\lambda}\left(\left(h_{i}, \alpha_{i}\right)\right)}{\sum_{i=1}^{n} \Gamma\left(\left(w_{i}, \beta_{i}\right)\right)}\right)^{1 / \lambda}\right),
\end{aligned}
$$


where $\left(h_{i}, \alpha_{i}\right),\left(w_{i}, \beta_{i}\right) \in \bar{S}\left(h_{i}, w_{i} \in \tilde{S} ; \alpha_{i}, \beta_{i} \in[-0.5\right.$, $0.5) ; i=1,2 \cdots, n)$, and $\lambda$ is a parameter in the interval $(0,+\infty)$.

Some desirable properties of the GE2T-WA operator are shown as follows.

Property 1. If $(h, \alpha),\left(h_{i}, \alpha_{i}\right),\left(\tilde{h}_{i}, \tilde{\alpha}_{i}\right) \in \bar{S}\left(h, h_{i}, \tilde{h}_{i} \in\right.$ $\left.\tilde{S} ; \alpha, \alpha_{i}, \tilde{\alpha}_{i} \in[-0.5,0.5) ; i=1,2 \cdots, n\right)$, then the following must be true:

(a) (Idempotence) For $\forall i$, if $\left(h_{i}, \alpha_{i}\right)=(h, \alpha)$, then:

$$
\operatorname{GE} 2 \mathrm{~T}-\mathrm{WA}\left(\left(h_{1}, \alpha_{1}\right),\left(h_{2}, \alpha_{2}\right), \cdots,\left(h_{n}, \alpha_{n}\right)\right)
$$

$$
=(h, \alpha) \text {. }
$$

(b) (Monotonicity) For $\forall i$, if $\left(\tilde{h}_{i}, \tilde{\alpha}_{i}\right) \leq\left(h_{i}, \alpha_{i}\right)$, then:

$$
\begin{aligned}
& \operatorname{GE} 2 \mathrm{~T}-\mathrm{WA}\left(\left(\tilde{h}_{1}, \tilde{\alpha}_{1}\right),\left(\tilde{h}_{2}, \tilde{\alpha}_{2}\right), \cdots,\left(\tilde{h}_{n}, \tilde{\alpha}_{n}\right)\right) \\
& \quad \leq \operatorname{GE} 2 \mathrm{~T}-\mathrm{WA}\left(\left(h_{1}, \alpha_{1}\right),\left(h_{2}, \alpha_{2}\right), \cdots,\left(h_{n}, \alpha_{n}\right)\right) .
\end{aligned}
$$

(c) (Boundedness)

$$
\begin{aligned}
& \min \left\{\left(h_{i}, \alpha_{i}\right)\right\} \\
& \quad \leq \operatorname{GE} 2 \mathrm{~T}-\mathrm{WA}\left(\left(h_{1}, \alpha_{1}\right),\left(h_{2}, \alpha_{2}\right), \cdots,\left(h_{n}, \alpha_{n}\right)\right) \\
& \quad \leq \max \left\{\left(h_{i}, \alpha_{i}\right)\right\} .
\end{aligned}
$$

\section{Proof.}

(a) For $\forall i$, if $\left(h_{i}, \alpha_{i}\right)=(h, \alpha)$, then:

$$
\begin{aligned}
& \operatorname{GE2T-WA}\left(\left(h_{1}, \alpha_{1}\right),\left(h_{2}, \alpha_{2}\right), \cdots,\left(h_{n}, \alpha_{n}\right)\right) \\
& =\Gamma^{-1}\left(\left(\sum_{i=1}^{n} \frac{\Gamma\left(\left(w_{i}, \beta_{i}\right)\right) \Gamma^{\lambda}\left(\left(h_{i}, \alpha_{i}\right)\right)}{\sum_{i=1}^{n} \Gamma\left(\left(w_{i}, \beta_{i}\right)\right)}\right)^{1 / \lambda}\right) \\
& =\Gamma^{-1}\left(\left(\sum_{i=1}^{n} \frac{\Gamma\left(\left(w_{i}, \beta_{i}\right)\right) \Gamma^{\lambda}((h, \alpha))}{\sum_{i=1}^{n} \Gamma\left(\left(w_{i}, \beta_{i}\right)\right)}\right)^{1 / \lambda}\right) \\
& =\Gamma^{-1}\left(\left(\Gamma^{\lambda}((h, \alpha))\right)^{1 / \lambda}\right)=(h, \alpha) .
\end{aligned}
$$

(b) For $\forall i$, if $\left(\tilde{h}_{i}, \tilde{\alpha}_{i}\right) \leq\left(h_{i}, \alpha_{i}\right), \Gamma\left(\left(\tilde{h}_{i}, \tilde{\alpha}_{i}\right)\right) \leq$ $\Gamma\left(\left(h_{i}, \alpha_{i}\right)\right)$ can be obtained according to Theorem 7. Therefore:

$$
\begin{aligned}
\sum_{i=1}^{n} & \frac{\Gamma\left(\left(w_{i}, \beta_{i}\right)\right) \Gamma^{\lambda}\left(\left(\tilde{h}_{i}, \tilde{\alpha}_{i}\right)\right)}{\sum_{i=1}^{n} \Gamma\left(\left(w_{i}, \beta_{i}\right)\right)} \\
& \leq \sum_{i=1}^{n} \frac{\Gamma\left(\left(w_{i}, \beta_{i}\right)\right) \Gamma^{\lambda}\left(\left(h_{i}, \alpha_{i}\right)\right)}{\sum_{i=1}^{n} \Gamma\left(\left(w_{i}, \beta_{i}\right)\right)} .
\end{aligned}
$$

According to Theorem 7, the following can be obtained:

$$
\begin{gathered}
\Gamma^{-1}\left(\left(\sum_{i=1}^{n} \frac{\Gamma\left(\left(w_{i}, \beta_{i}\right)\right) \Gamma^{\lambda}\left(\left(\tilde{h}_{i}, \tilde{\alpha}_{i}\right)\right)}{\sum_{i=1}^{n} \Gamma\left(\left(w_{i}, \beta_{i}\right)\right)}\right)^{1 / \lambda}\right) \\
\leq \Gamma^{-1}\left(\left(\sum_{i=1}^{n} \frac{\Gamma\left(\left(w_{i}, \beta_{i}\right)\right) \Gamma^{\lambda}\left(\left(h_{i}, \alpha_{i}\right)\right)}{\sum_{i=1}^{n} \Gamma\left(\left(w_{i}, \beta_{i}\right)\right)}\right)^{1 / \lambda}\right) .
\end{gathered}
$$

Therefore:

$$
\begin{gathered}
\operatorname{GE} 2 \mathrm{~T}-W A\left(\left(\tilde{h}_{1}, \tilde{\alpha}_{1}\right),\left(\tilde{h}_{2}, \tilde{\alpha}_{2}\right), \cdots,\left(\tilde{h}_{n}, \tilde{\alpha}_{n}\right)\right) \\
\leq \operatorname{GE} 2 \mathrm{~T}-W A\left(\left(h_{1}, \alpha_{1}\right),\left(h_{2}, \alpha_{2}\right), \cdots,\left(h_{n}, \alpha_{n}\right)\right) .
\end{gathered}
$$

(c) Since $\min \left\{\left(h_{i}, \alpha_{i}\right)\right\} \leq\left(h_{i}, \alpha_{i}\right)$ and $\max \left\{\left(h_{i}, \alpha_{i}\right)\right\} \geq$ $\left(h_{i}, \alpha_{i}\right)$, according to Property $1(\mathrm{a})$ :

$$
\begin{gathered}
\min \left\{\left(h_{i}, \alpha_{i}\right)\right\}=\text { GE2T-WA }\left(\min \left\{\left(h_{i}, \alpha_{i}\right)\right\},\right. \\
\left.\min \left\{\left(h_{i}, \alpha_{i}\right)\right\}, \cdots, \min \left\{\left(h_{i}, \alpha_{i}\right)\right\}\right),
\end{gathered}
$$

can be obtained and according to Property $1(\mathrm{~b})$ : GE2T-WA $\left(\min \left\{\left(h_{i}, \alpha_{i}\right)\right\}, \min \left\{\left(h_{i}, \alpha_{i}\right)\right\}, \cdots\right.$,

$$
\begin{aligned}
& \left.\min \left\{\left(h_{i}, \alpha_{i}\right)\right\}\right) \leq \operatorname{GE} 2 \mathrm{~T}-\mathrm{WA}\left(\left(h_{1}, \alpha_{1}\right),\left(h_{2}, \alpha_{2}\right),\right. \\
& \left.\cdots,\left(h_{n}, \alpha_{n}\right)\right),
\end{aligned}
$$

can also be obtained.

Similarly:

$$
\begin{aligned}
\operatorname{GE} 2 \mathrm{~T}-\mathrm{WA} & \left(\left(h_{1}, \alpha_{1}\right),\left(h_{2}, \alpha_{2}\right), \cdots,\left(h_{n}, \alpha_{n}\right)\right) \\
\leq & \max \left\{\left(h_{i}, \alpha_{i}\right)\right\} .
\end{aligned}
$$

Therefore,

$$
\begin{aligned}
\min & \left\{\left(h_{i}, \alpha_{i}\right)\right\} \\
& \leq \operatorname{GE} 2 \mathrm{~T}-\mathrm{WA}\left(\left(h_{1}, \alpha_{1}\right),\left(h_{2}, \alpha_{2}\right), \cdots,\left(h_{n}, \alpha_{n}\right)\right) \\
& \leq \max \left\{\left(h_{i}, \alpha_{i}\right)\right\} .
\end{aligned}
$$

Definition 9. Let $\left(h_{i}, \alpha_{i}\right)(i=1,2 \cdots, n)$ be a set of $n$ 2-tuples which has an associated weight vector $w=$ $\left(\left(w_{1}, \beta_{1}\right),\left(w_{2}, \beta_{2}\right), \cdots,\left(w_{n}, \beta_{n}\right)\right)$. Then, the GE2TOWA operator can be defined as follows:

$$
\begin{aligned}
& \operatorname{GE2T-OWA}: \bar{S}^{n} \rightarrow \bar{S}, \\
& \operatorname{GE2T-OWA}\left(\left(h_{1}, \alpha_{1}\right),\left(h_{2}, \alpha_{2}\right), \cdots,\left(h_{n}, \alpha_{n}\right)\right) \\
& =\Gamma^{-1}\left(\left(\sum_{i=1}^{n} \frac{\Gamma\left(\left(w_{i}, \beta_{i}\right)\right) \Gamma^{\lambda}\left(\left(h_{\sigma(i)}, \alpha_{\sigma(i))}\right)\right.}{\sum_{i=1}^{n} \Gamma\left(\left(w_{i}, \beta_{i}\right)\right)}\right)^{1 / \lambda}\right),
\end{aligned}
$$


where $\left(h_{i}, \alpha_{i}\right),\left(h_{\sigma(i)}, \alpha_{\sigma(i)}\right),\left(w_{i}, \beta_{i}\right) \in \bar{S}\left(h_{i}, h_{\sigma(i)}, w_{i} \in\right.$ $\left.\tilde{S} ; \alpha_{i}, \alpha_{\sigma(i)}, \beta_{i} \in[-0.5,0.5) ; i=1,2 \cdots, n\right), \lambda$ is a parameter in the interval $(0,+\infty)$ and a permutation of $(1,2, \cdots, n)$ is $(\sigma(1), \sigma(2), \cdots, \sigma(n))$, which satisfies the inequality $\left(h_{\sigma(i)}, \alpha_{\sigma(i)}\right) \geq\left(h_{\sigma(i+1)}, \alpha_{\sigma(i+1)}\right)$ for $\forall i \in$ $\{1,2, \cdots, n-1\}$.

Some properties of the GE2T-OWA operator are shown below:

Property 2. If $(h, \alpha),\left(h_{i}, \alpha_{i}\right),\left(\tilde{h}_{i}, \tilde{\alpha}_{i}\right),\left(\bar{h}_{i}, \bar{\alpha}_{i}\right) \in$ $\bar{S}\left(h, h_{i}, \tilde{h}_{i}, \bar{h}_{i} \in \tilde{S} ; \alpha, \alpha_{i}, \tilde{\alpha}_{i}, \bar{\alpha}_{i} \in[-0.5,0.5) ; i=\right.$ $1,2 \cdots, n)$, then the following must be true:

(a) (Idempotence) For $\forall i$, if $\left(h_{i}, \alpha_{i}\right)=(h, \alpha)$, then

$$
\begin{gathered}
\operatorname{GE2T-OWA}\left(\left(h_{1}, \alpha_{1}\right),\left(h_{2}, \alpha_{2}\right), \cdots,\left(h_{n}, \alpha_{n}\right)\right) \\
=(h, \alpha) .
\end{gathered}
$$

(b) (Monotonicity) For $\forall i$, if $\left(\tilde{h}_{i}, \tilde{\alpha}_{i}\right) \leq\left(h_{i}, \alpha_{i}\right)$, then:

$$
\begin{gathered}
\operatorname{GE} 2 \mathrm{~T}-O W A\left(\left(\tilde{h}_{1}, \tilde{\alpha}_{1}\right),\left(\tilde{h}_{2}, \tilde{\alpha}_{2}\right) \cdots,\left(\tilde{h}_{n}, \tilde{\alpha}_{n}\right)\right) \\
\quad \leq \operatorname{GE} 2 \mathrm{~T}-\mathrm{OWA}\left(\left(h_{1}, \alpha_{1}\right),\left(h_{2}, \alpha_{2}\right), \cdots,\left(h_{n}, \alpha_{n}\right)\right) .
\end{gathered}
$$

(c) (Boundedness)

$$
\begin{aligned}
\min & \left\{\left(h_{i}, \alpha_{i}\right)\right\} \\
& \leq \operatorname{GE} 2 \mathrm{~T}-\mathrm{OWA}\left(\left(h_{1}, \alpha_{1}\right),\left(h_{2}, \alpha_{2}\right), \cdots,\left(h_{n}, \alpha_{n}\right)\right) \\
& \leq \max \left\{\left(h_{i}, \alpha_{i}\right)\right\} .
\end{aligned}
$$

(d) (Commutativity)

If $\left(\left(\bar{h}_{1}, \bar{\alpha}_{1}\right),\left(\bar{h}_{2}, \bar{\alpha}_{2}\right), \cdots,\left(\bar{h}_{n}, \bar{\alpha}_{n}\right)\right)$ is a permutation of $\left(\left(h_{1}, \alpha_{1}\right),\left(h_{2}, \alpha_{2}\right), \cdots,\left(h_{n}, \alpha_{n}\right)\right)$, then:

$$
\begin{aligned}
& \operatorname{GE} 2 \text { T-OWA }\left(\left(\bar{h}_{1}, \bar{\alpha}_{1}\right),\left(\bar{h}_{2}, \bar{\alpha}_{2}\right), \cdots,\left(\bar{h}_{n}, \bar{\alpha}_{n}\right)\right) \\
& \quad=\operatorname{GE2T-OWA}\left(\left(h_{1}, \alpha_{1}\right),\left(h_{2}, \alpha_{2}\right), \cdots,\left(h_{n}, \alpha_{n}\right)\right) .
\end{aligned}
$$

Proof. For proof of items (a), (b), and (c), one can refer to Property 1, and item (d) can be obtained as follows:

since $\left(\left(\bar{h}_{1}, \bar{\alpha}_{1}\right),\left(\bar{h}_{2}, \bar{\alpha}_{2}\right), \cdots,\left(\bar{h}_{n}, \bar{\alpha}_{n}\right)\right)$ is a permutation of $\left(\left(h_{1}, \alpha_{1}\right),\left(h_{2}, \alpha_{2}\right), \cdots,\left(h_{n}, \alpha_{n}\right)\right)$,

$$
\begin{aligned}
& \operatorname{GE} 2 \mathrm{~T} \text {-OWA }\left(\left(\bar{h}_{1}, \bar{\alpha}_{1}\right),\left(\bar{h}_{2}, \bar{\alpha}_{2}\right), \cdots,\left(\bar{h}_{n}, \bar{\alpha}_{n}\right)\right) \\
& =\Gamma^{-1}\left(\left(\sum_{i=1}^{n} \frac{\Gamma\left(\left(w_{i}, \beta_{i}\right)\right) \Gamma^{\lambda}\left(\left(h_{\sigma(i)}, \alpha_{\sigma(i)}\right)\right)}{\sum_{i=1}^{n} \Gamma\left(\left(w_{i}, \beta_{i}\right)\right)}\right)^{1 / \lambda}\right) \\
& =\operatorname{GE} 2 \operatorname{T-OWA}\left(\left(h_{1}, \alpha_{1}\right),\left(h_{2}, \alpha_{2}\right), \cdots,\left(h_{n}, \alpha_{n}\right)\right) .
\end{aligned}
$$

Definition 10. Let $\left(h_{i}, \alpha_{i}\right)(i=1,2 \cdots, n)$ be a set of $n$ 2-tuples which has an associated weight vector $w=$ $\left(\left(w_{1}, \beta_{1}\right),\left(w_{2}, \beta_{2}\right), \cdots,\left(w_{n}, \beta_{n}\right)\right)$. Then, the GE2TWG operator can be defined as follows:

$$
\begin{aligned}
& \text { GE2T-WG }: \bar{S}^{n} \rightarrow \bar{S}, \\
& \begin{aligned}
\text { GE2T - WG }\left(\left(h_{1}, \alpha_{1}\right),\left(h_{2}, \alpha_{2}\right), \cdots,\left(h_{n}, \alpha_{n}\right)\right) \\
=\Gamma^{-1}\left(\prod_{i=1}^{n}\left(\Gamma\left(\left(h_{i}, \alpha_{i}\right)\right)\right)^{\frac{\Gamma\left(\left(w_{i}, \beta_{i}\right)\right)}{\sum_{i=1}^{n} \Gamma\left(\left(w_{i}, \beta_{i}\right)\right)}}\right),
\end{aligned}
\end{aligned}
$$

where:

$$
\begin{aligned}
\left(h_{i}, \alpha_{i}\right),\left(w_{i}, \beta_{i}\right) & \in \bar{S}\left(h_{i}, w_{i} \in \tilde{S} ; \alpha_{i}, \beta_{i}\right. \\
& \in[-0.5,0.5) ; i=1,2 \cdots, n) .
\end{aligned}
$$

Some properties of the GE2T-WG operator are shown as follows.

Property 3. If $(h, \alpha),\left(h_{i}, \alpha_{i}\right),\left(\tilde{h}_{i}, \tilde{\alpha}_{i}\right) \in \bar{S}\left(h, h_{i}, \tilde{h}_{i} \in\right.$ $\left.\tilde{S} ; \alpha, \alpha_{i}, \tilde{\alpha}_{i} \in[-0.5,0.5) ; i=1,2 \cdots, n\right)$, then the following must be true.

(a) (Idempotence) For $\forall i$, if $\left(h_{i}, \alpha_{i}\right)=(h, \alpha)$, then:

$$
\operatorname{GE} 2 \mathrm{~T}-\mathrm{WG}\left(\left(h_{1}, \alpha_{1}\right),\left(h_{2}, \alpha_{2}\right), \cdots,\left(h_{n}, \alpha_{n}\right)\right)=(h, \alpha) .
$$

(b) (Monotonicity) For $\forall i$, if $\left(\tilde{h}_{i}, \tilde{\alpha}_{i}\right) \leq\left(h_{i}, \alpha_{i}\right)$, then:

$$
\begin{gathered}
\operatorname{GE} 2 \mathrm{~T}-\mathrm{WG}\left(\left(\tilde{h}_{1}, \tilde{\alpha}_{1}\right),\left(\tilde{h}_{2}, \tilde{\alpha}_{2}\right), \cdots,\left(\tilde{h}_{n}, \tilde{\alpha}_{n}\right)\right) \\
\leq \operatorname{GE} 2 \mathrm{~T}-\mathrm{WG}\left(\left(h_{1}, \alpha_{1}\right),\left(h_{2}, \alpha_{2}\right), \cdots,\left(h_{n}, \alpha_{n}\right)\right) .
\end{gathered}
$$

(c) (Boundedness)

$$
\begin{aligned}
\min & \left\{\left(h_{i}, \alpha_{i}\right)\right\} \\
& \leq \operatorname{GE} 2 \mathrm{~T}-\mathrm{WG}\left(\left(h_{1}, \alpha_{1}\right),\left(h_{2}, \alpha_{2}\right), \cdots,\left(h_{n}, \alpha_{n}\right)\right) \\
& \leq \max \left\{\left(h_{i}, \alpha_{i}\right)\right\} .
\end{aligned}
$$

For proof of Property 3, one can refer to Property 1.

Definition 11. Let $\left(h_{i}, \alpha_{i}\right)=(1,2, \ldots, n)$ be a set of $n$ 2-tuples which has an associated weight vector $w=$ $\left(\left(w_{1}, \beta_{1}\right),\left(w_{2}, \beta_{2}\right), \cdots,\left(w_{n}, \beta_{n}\right)\right)$. Then, the GE2TWG operator can be defined as follows:

$$
\begin{aligned}
& \text { GE2T-OWG }: \bar{S}^{n} \rightarrow \bar{S}, \\
& \text { GE2T-OWG }\left(\left(h_{1}, \alpha_{1}\right),\left(h_{2}, \alpha_{2}\right), \cdots,\left(h_{n}, \alpha_{n}\right)\right) \\
& \left.=\Gamma^{-1}\left(\prod_{i=1}^{n}\left(\Gamma\left(\left(h_{\sigma(i)}, \alpha_{\sigma(i)}\right)\right)\right)\right)^{\frac{\Gamma\left(\left(w_{i}, \beta_{i}\right)\right)}{\sum_{i=1}^{n} \Gamma\left(\left(w_{i}, \beta_{i}\right)\right)}}\right),
\end{aligned}
$$


where:

$$
\begin{aligned}
& \left(h_{i}, \alpha_{i}\right),\left(h_{\sigma(i)}, \alpha_{\sigma(i)}\right),\left(w_{i}, \beta_{i}\right) \in \bar{S}\left(h_{i}, h_{\sigma(i)}, w_{i}\right. \\
& \left.\quad \in \tilde{S} ; \alpha_{i}, \alpha_{\sigma(i)}, \beta_{i} \in[-0.5,0.5) ; i=1,2 \cdots, n\right),
\end{aligned}
$$

and a permutation of $(1,2, \cdots, n)$ is $(\sigma(1), \sigma(2)$, $\cdots, \sigma(n))$, which satisfies the inequality $\left(h_{\sigma(i)}, \alpha_{\sigma(i)}\right) \geq$ $\left(h_{\sigma(i+1)}, \alpha_{\sigma(i+1)}\right)$ for $\forall i \in\{1,2, \cdots, n-1\}$.

Some properties of the GE2T-OWG operator are shown as follows:

Property 4. If $(h, \alpha),\left(h_{i}, \alpha_{i}\right),\left(\tilde{h}_{i}, \tilde{\alpha}_{i}\right),\left(\bar{h}_{i}, \bar{\alpha}_{i}\right) \quad \in$ $\bar{S}\left(h, h_{i}, \tilde{h}_{i}, \bar{h}_{i} \in \tilde{S} ; \alpha, \alpha_{i}, \tilde{\alpha}_{i}, \bar{\alpha}_{i} \in[-0.5,0.5) ; i=\right.$ $1,2 \cdots, n)$, then the following must be true:

(a) (Idempotence) For $\forall i$, if $\left(h_{i}, \alpha_{i}\right)=(h, \alpha)$, then:

$$
\begin{gathered}
\operatorname{GE2T-OWG}\left(\left(h_{1}, \alpha_{1}\right),\left(h_{2}, \alpha_{2}\right), \cdots,\left(h_{n}, \alpha_{n}\right)\right) \\
=(h, \alpha) .
\end{gathered}
$$

(b) (Monotonicity) For $\forall i$, if $\left(\tilde{h}_{i}, \tilde{\alpha}_{i}\right) \leq\left(h_{i}, \alpha_{i}\right)$, then:

$$
\begin{aligned}
& \operatorname{GE} 2 T-O W G\left(\left(\tilde{h}_{1}, \tilde{\alpha}_{1}\right),\left(\tilde{h}_{2}, \tilde{\alpha}_{2}\right), \cdots,\left(\tilde{h}_{n}, \tilde{\alpha}_{n}\right)\right) \\
& \leq \operatorname{GE} 2 \mathrm{~T}-O W G\left(\left(h_{1}, \alpha_{1}\right),\left(h_{2}, \alpha_{2}\right), \cdots,\left(h_{n}, \alpha_{n}\right)\right) .
\end{aligned}
$$

(c) (Boundedness)

$$
\begin{aligned}
\min & \left\{\left(h_{i}, \alpha_{i}\right)\right\} \\
& \leq \operatorname{GE} 2 \mathrm{~T}-\mathrm{OWG}\left(\left(h_{1}, \alpha_{1}\right),\left(h_{2}, \alpha_{2}\right), \cdots,\left(h_{n}, \alpha_{n}\right)\right) \\
& \leq \max \left\{\left(h_{i}, \alpha_{i}\right)\right\} .
\end{aligned}
$$

(d) (Commutativity) If $\left(\left(\bar{h}_{1}, \bar{\alpha}_{1}\right),\left(\bar{h}_{2}, \bar{\alpha}_{2}\right), \cdots,\left(\bar{h}_{n}\right.\right.$, $\left.\left.\bar{\alpha}_{n}\right)\right)$ is a permutation of $\left(\left(h_{1}, \alpha_{1}\right),\left(h_{2}, \alpha_{2}\right), \cdots\right.$, $\left.\left(h_{n}, \alpha_{n}\right)\right)$, then:

$$
\begin{aligned}
& \operatorname{GE2T-OWG}\left(\left(\bar{h}_{1}, \bar{\alpha}_{1}\right),\left(\bar{h}_{2}, \bar{\alpha}_{2}\right), \cdots,\left(\bar{h}_{n}, \bar{\alpha}_{n}\right)\right) \\
& \quad=\operatorname{GE} 2 \mathrm{T-OWG}\left(\left(h_{1}, \alpha_{1}\right),\left(h_{2}, \alpha_{2}\right), \cdots,\left(h_{n}, \alpha_{n}\right)\right) .
\end{aligned}
$$

For proof of Property 4, one can refer to Property 2.

Example 9. Use the data of Example 1, let $\bar{S}_{3}=$ $\left\{\left(s_{0}, 0.2\right),\left(s_{2},-0.2\right),\left(s_{3}, 0\right),\left(s_{4}, 0.3\right),\left(s_{5}, 0\right)\right\}$ be a set of four 2-tuples, its associated weight vector be $w_{3}=$ $\left(\left(s_{2}, 0.1\right),\left(s_{0}, 0.1\right),\left(s_{1}, 0.3\right),\left(s_{3},-0.1\right),\left(s_{2}, 0\right)\right)$, the subjective distances of the adjacent evaluation values be $\operatorname{dist}\left(s_{j-1}, s_{j}\right)=1 . a=1.4, \lambda=1$ and:

$$
\begin{aligned}
\Gamma((\hat{s}, \hat{\alpha}))= & \Gamma\left(\left(s_{2}, 0.1\right)\right)+\Gamma\left(\left(s_{0}, 0.1\right)\right)+\Gamma\left(\left(s_{1}, 0.3\right)\right) \\
& +\Gamma\left(\left(s_{3},-0.1\right)\right)+\Gamma\left(\left(s_{2}, 0\right)\right)
\end{aligned}
$$

Therefore, the results, shown in Box I, are true.

\section{A 2-tuple linguistic MCGDM method based on the proposed 2-tuple linguistic operators}

In this section, the GE2T-WA and GE2T-OWA operators, or the GE2T-WG and GE2T-OWG operators, are applied to MCGDM problems based on linguistic information.

For an MCGDM problem with linguistic information, let $S$ be a linguistic term set and $\tilde{S}$ be an extended linguistic term set, $D=\left\{D_{1}, D_{2}, \cdots, D_{p}\right\}$ be the set of decision-makers and $\tau=\left(\tau_{1}, \tau_{2}, \cdots, \tau_{p}\right)$ be the weight vector of decision-makers, where $\tau_{k} \in$ $S(k=1,2, \cdots, p)$, and let $A=\left\{A_{1}, A_{2}, \cdots, A_{m}\right\}$ be the set of alternatives and $C=\left\{C_{1}, C_{2}, \cdots, C_{n}\right\}$ be the set of criteria whose associated weight vector is $w=\left(w_{1}, w_{2}, \cdots, w_{n}\right)$, where $w_{j} \in S, j=$ $1,2, \cdots, n$. The decision-maker $D_{k}(k=1,2, \cdots, p)$ evaluates the alternative $A_{i}(i=1,2, \cdots, m)$ with respect to the criteria $C_{j}(j=1,2, \cdots, n)$ to get $h_{i j}^{k}(i=$ $1,2, \cdots, m, j=1,2, \cdots, n)$, where $h_{i j}^{k} \in S$, and then the decision matrix $R_{k}=\left(h_{i j}^{k}\right)_{m \times n}$ is obtained. A method of determining the ranking of the alternatives is introduced in the following subsection.

The decision-making procedure is as follows:

Step 1. Normalize the decision matrices.

Normalize the decision-making information $h_{i j}^{k}$ in the matrix $R_{k}=\left(h_{i j}^{k}\right)_{m \times n}$. The criteria can be classified into the benefit type and the cost type. For the benefit-type criterion, the form of the linguistic information changes nothing; but for the cost-type criterion, the linguistic negation operator is used.

The normalization of the decision matrices can be represented as follows:

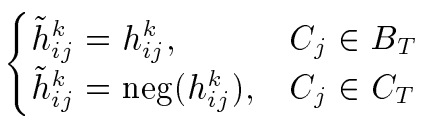

where $B_{T}$ is the set of benefit-type criteria and $C_{T}$ is the set of cost-type criteria.

The normalized decision matrices are denoted as $\bar{R}_{k}=\left(\tilde{h}_{i j}^{k}\right)_{m \times n}$;

Step 2. Convert the linguistic information into 2 tuples.

Convert the decision-making information $\tilde{h}_{i j}^{k}$ in the matrix $\bar{R}_{k}=\left(\tilde{h}_{i j}^{k}\right)_{m \times n}$ to $\left(\tilde{h}_{i j}^{k}, 0\right)$ for obtaining the matrix $\tilde{R}_{k}\left(\left(\tilde{h}_{i j}^{k}, 0\right)\right)_{m \times n}$; convert the weight vector of decision-makers $\tau=\left(\tau_{1}, \tau_{2}, \cdots, \tau_{p}\right)$ to $\tilde{\tau}=$ $\left(\left(\tau_{1}, 0\right),\left(\tau_{2}, 0\right), \cdots,\left(\tau_{p}, 0\right)\right)$; and convert the weight vector of criteria $w=\left(w_{1}, w_{2}, \cdots, w_{n}\right)$ to $\tilde{w}=$ $\left(\left(w_{1}, 0\right),\left(w_{2}, 0\right), \cdots,\left(w_{n}, 0\right)\right)$;

Step 3. Aggregate all the values of each alternative on each criterion to get the collective linguistic 2tuple values.

Based on Definition 8 or Definition 10, the collective linguistic 2-tuple values $\left(h_{i}^{k}, \alpha_{i}^{k}\right)\left(h_{i}^{k} \in \tilde{S}, \alpha_{i}^{k} \in\right.$ 


$$
\begin{aligned}
\mathrm{GE} 2 \mathrm{~T} & -\mathrm{WA}\left(\left(s_{0}, 0.2\right),\left(s_{2},-0.2\right),\left(s_{3}, 0\right),\left(s_{4}, 0.3\right),\left(s_{5}, 0\right)\right) \\
& =\Gamma^{-1}\left(\frac{\Gamma\left(\left(s_{2}, 0.1\right)\right) \Gamma\left(\left(s_{0}, 0.2\right)\right)+\Gamma\left(\left(s_{0}, 0.1\right)\right) \Gamma\left(\left(s_{2},-0.2\right)\right)+\Gamma\left(\left(s_{1}, 0.3\right)\right) \Gamma\left(\left(s_{3}, 0\right)\right)}{\Gamma((\hat{s}, \hat{\alpha}))}\right. \\
& \left.+\frac{\Gamma\left(\left(s_{3},-0.1\right)\right) \Gamma\left(\left(s_{4}, 0.3\right)\right)+\Gamma\left(\left(s_{2}, 0\right)\right) \Gamma\left(\left(s_{5}, 0\right)\right)}{\Gamma((\hat{s}, \hat{\alpha}))}\right)=\Gamma^{-1}(0.543)=\overline{\operatorname{SNS}}^{-1}(0.411)=\left(s_{3}, 0.411\right),
\end{aligned}
$$

$\operatorname{GE} 2 \mathrm{~T}-\mathrm{OWA}\left(\left(s_{0}, 0.2\right),\left(s_{2},-0.2\right),\left(s_{3}, 0\right),\left(s_{4}, 0.3\right),\left(s_{5}, 0\right)\right)$

$$
\begin{aligned}
& =\Gamma^{-1}\left(\frac{\Gamma\left(\left(s_{2}, 0.1\right)\right) \Gamma\left(\left(s_{5}, 0\right)\right)+\Gamma\left(\left(s_{0}, 0.1\right)\right) \Gamma\left(\left(s_{4}, 0.3\right)\right)+\Gamma\left(\left(s_{1}, 0.3\right)\right) \Gamma\left(\left(s_{3}, 0\right)\right)}{\Gamma((\hat{s}, \hat{\alpha}))}\right. \\
& \left.+\frac{\Gamma\left(\left(s_{3},-0.1\right)\right) \Gamma\left(\left(s_{2},-0.2\right)\right)+\Gamma\left(\left(s_{2}, 0\right)\right) \Gamma\left(\left(s_{0}, 0.2\right)\right)}{\Gamma((\hat{s}, \hat{\alpha}))}\right)=\Gamma^{-1}(0.372)=\overline{\mathrm{SNS}}^{-1}(-1.099)=\left(s_{2},-0.099\right),
\end{aligned}
$$

$\mathrm{GE} 2 \mathrm{~T}-\mathrm{WG}\left(\left(s_{0}, 0.2\right),\left(s_{2},-0.2\right),\left(s_{3}, 0\right),\left(s_{4}, 0.3\right),\left(s_{5}, 0\right)\right)=\Gamma^{-1}\left(\left(\Gamma\left(\left(s_{0}, 0.2\right)\right)\right)^{\frac{\Gamma\left(\left(s_{2}, 0.1\right)\right)}{\Gamma((\hat{s}, \hat{\alpha}))}}\right.$

$$
\begin{aligned}
& \left.\times\left(\Gamma\left(\left(s_{2},-0.2\right)\right)\right)^{\frac{\Gamma\left(\left(s_{0}, 0.1\right)\right)}{\Gamma((\hat{s}, \hat{\alpha}))}} \times\left(\Gamma\left(\left(s_{3}, 0\right)\right)\right)^{\frac{\Gamma\left(\left(s_{1}, 0.3\right)\right)}{\Gamma((\hat{s}, \hat{\alpha}))}} \times\left(\Gamma\left(\left(s_{4}, 0.3\right)\right)\right)^{\frac{\Gamma\left(\left(s_{3},-0.1\right)\right)}{\Gamma((\hat{\beta}, \hat{\alpha}))}} \times\left(\Gamma\left(\left(s_{5}, 0\right)\right)\right)^{\frac{\Gamma\left(\left(s_{2}, 0\right)\right)}{\Gamma((\hat{s}, \hat{\alpha}))}}\right) \\
& =\Gamma^{-1}(0.376)=\overline{\operatorname{SNS}}^{-1}(-1.065)=\left(s_{2},-0.065\right)
\end{aligned}
$$

$\operatorname{GE} 2 \mathrm{~T}-\mathrm{OWG}\left(\left(s_{0}, 0.2\right),\left(s_{2},-0.2\right),\left(s_{3}, 0\right),\left(s_{4}, 0.3\right),\left(s_{5}, 0\right)\right)=\Gamma^{-1}\left(\left(\Gamma\left(\left(s_{5}, 0\right)\right)\right)^{\frac{\Gamma\left(\left(s_{2}, 0.1\right)\right)}{\Gamma((\hat{s}, \hat{\alpha}))}}\right.$

$$
\begin{aligned}
& \left.\times\left(\Gamma\left(\left(s_{4}, 0.3\right)\right)\right)^{\frac{\Gamma\left(\left(s_{0}, 0.1\right)\right)}{\Gamma((\hat{s}, \hat{\alpha}))}} \times\left(\Gamma\left(\left(s_{3}, 0\right)\right)\right)^{\frac{\Gamma\left(\left(s_{1}, 0.3\right)\right)}{\Gamma((\hat{s}, \hat{\alpha}))}} \times\left(\Gamma\left(\left(s_{2},-0.2\right)\right)\right)^{\frac{\Gamma\left(\left(s_{3},-0.1\right)\right)}{\Gamma((\hat{s}, \hat{\alpha}))}} \times\left(\Gamma\left(\left(s_{0}, 0.2\right)\right)\right)^{\frac{\Gamma\left(\left(s_{2}, 0\right)\right)}{\Gamma((\hat{s}, \hat{\alpha}))}}\right) \\
& =\Gamma^{-1}(0.233)=\overline{\mathrm{SNS}}^{-1}(-1.958)=\left(s_{1}, 0.042\right) .
\end{aligned}
$$

$[-0.5,0.5))$ or $\left(\tilde{h}_{i}^{k}, \tilde{\alpha}_{i}^{k}\right)\left(\tilde{h}_{i}^{k} \in \tilde{S}, \tilde{\alpha}_{i}^{k} \in[-0.5,0.5)\right)$ can be obtained by using the GE2T-WA or GE2TWG operator to aggregate all the values of each alternative on each criterion as follows:

$$
\begin{array}{r}
\left(h_{i}^{k}, \alpha_{i}^{k}\right)=\operatorname{GE} 2 \mathrm{~T}-\mathrm{WA}\left(\left(\tilde{h}_{i 1}^{k}, 0\right),\left(\tilde{h}_{i 2}^{k}, 0\right), \cdots,\left(\tilde{h}_{i n}^{k}, 0\right)\right) \\
=\Gamma^{-1}\left(\left(\sum_{j=1}^{n} \frac{\Gamma\left(\left(w_{j}, 0\right)\right) \Gamma^{\lambda}\left(\left(\tilde{h}_{i j}^{k}, 0\right)\right)}{\sum_{j=1}^{n} \Gamma\left(\left(w_{j}, 0\right)\right)}\right)^{1 / \lambda}\right) .
\end{array}
$$

or

$$
\begin{aligned}
\left(\tilde{h}_{i}^{k}, \tilde{\alpha}_{i}^{k}\right) & =\operatorname{GE} 2 \mathrm{~T}-\mathrm{WG}\left(\left(\tilde{h}_{i 1}^{k}, 0\right),\left(\tilde{h}_{i 2}^{k}, 0\right), \cdots,\left(\tilde{h}_{i n}^{k}, 0\right)\right) \\
& \left.=\Gamma^{-1}\left(\prod_{j=1}^{n}\left(\Gamma\left(\left(\tilde{h}_{i j}^{k}, 0\right)\right)\right)\right)^{\frac{\Gamma\left(\left(w_{j}, 0\right)\right)}{\sum_{j=1}^{n} \Gamma\left(\left(w_{j}, 0\right)\right)}}\right) .
\end{aligned}
$$

Subsequently, the collective preference matrix $P=$ $\left(\left(h_{i}^{k}, \alpha_{i}^{k}\right)\right)_{p \times m}$ or $\tilde{P}=\left(\left(\tilde{h}_{i}^{k}, \tilde{\alpha}_{i}^{k}\right)\right)_{p \times m}$ can be obtained;
Step 4. Aggregate the decisions in the matrix $P$ or $\tilde{P}$ to derive the overall values of alternative $A_{i}$.

Based on Definition 9 or Definition 11, the overall values $\left(h_{i}, \alpha_{i}\right)\left(h_{i} \in \tilde{S}, \alpha_{i} \in[-0.5,0.5)\right)$ or $\left(\tilde{h}_{i}, \tilde{\alpha}_{i}\right)\left(\tilde{h}_{i} \in \tilde{S}, \tilde{\alpha}_{i} \in[-0.5,0.5)\right)$ of the alternative $A_{i}$ can be obtained by using the GE2T-OWA or GE2TOWG operators:

$$
\begin{array}{r}
\left(h_{i}, \alpha_{i}\right)=\operatorname{GE} 2 \mathrm{~T}-\mathrm{OWA}\left(\left(h_{i}^{1}, \alpha_{i}^{1}\right),\left(h_{i}^{2}, \alpha_{i}^{2}\right), \cdots,\left(h_{i}^{p}, \alpha_{i}^{p}\right)\right) \\
=\Gamma^{-1}\left(\left(\sum_{k=1}^{p} \frac{\Gamma\left(\left(\tau_{k}, 0\right)\right) \Gamma^{\lambda}\left(\left(h_{\sigma(j)}^{k}, \alpha_{\sigma(j)}^{k}\right)\right)}{\sum_{k=1}^{p} \Gamma\left(\left(\tau_{k}, 0\right)\right)}\right),\right.
\end{array}
$$

or:

$$
\begin{array}{r}
\left(\tilde{h}_{i}, \tilde{\alpha}_{i}\right)=\operatorname{GE} 2 \mathrm{~T}-\mathrm{OWG}\left(\left(\tilde{h}_{i}^{1}, \tilde{\alpha}_{i}^{1}\right),\left(\tilde{h}_{i}^{2}, \tilde{\alpha}_{i}^{2}\right), \cdots,\left(\tilde{h}_{i}^{p}, \tilde{\alpha}_{i}^{p}\right)\right) \\
\left.=\Gamma^{-1}\left(\prod_{k=1}^{p}\left(\Gamma\left(\left(\tilde{h}_{\sigma(j)}^{k}, \tilde{\alpha}_{\sigma(j)}^{k}\right)\right)\right)\right)_{k=1}^{\left.\frac{\Gamma\left(\left(\tau_{k}, 0\right)\right)}{\sum_{k=1}^{p} \Gamma\left(\left(\tau_{k}, 0\right)\right)}\right)}\right)
\end{array}
$$


where $\left(h_{\sigma(j)}^{k}, \alpha_{\sigma(j)}^{k}\right)$ is the $j$ th biggest 2 tuple in $\left(\left(\tilde{h}_{1}^{k}, \tilde{\alpha}_{1}^{k}\right),\left(\tilde{h}_{2}^{k}, \tilde{\alpha}_{2}^{k}\right), \cdots,\left(\tilde{h}_{m}^{k}, \tilde{\alpha}_{m}^{k}\right)\right)$ and $\left(\tilde{h}_{\sigma(j)}^{k}, \tilde{\alpha}_{\sigma(j)}^{k}\right)$ is the $j$ th biggest 2-tuple in $\left(\left(\tilde{h}_{1}^{k}, \tilde{\alpha}_{1}^{k}\right),\left(\tilde{h}_{2}^{k}, \tilde{\alpha}_{2}^{k}\right)\right.$, $\left.\cdots,\left(\tilde{h}_{m}^{k}, \tilde{\alpha}_{m}^{k}\right)\right)$;

Step 5. Rank the alternatives and select the best one.

The ranking of the alternative $A_{i}$ can be obtained according to Definition 3 , and thus the best one can be determined.

\section{An illustrative example}

In this section, an investment problem (adapted from [17]) is used to illustrate the proposed method.

A practical use of this method is demonstrated by choosing the best investment project for an investment company, which has a sum of money available for such a purpose. Five possible alternatives are $A_{i}(i=$ $1,2, \cdots, 5)$ whose criteria are $C_{j}(j=1,2,3,4)$, i.e. the risk, growth, social-political impact, and environmental impact factors. The linguistic term set is $S_{1}=\left\{s_{0}=\right.$ Extremely Poor (EP), $s_{1}=$ Very Poor (VP), $s_{2}=$ Poor $(\mathrm{P}), s_{3}=\operatorname{Medium}(\mathrm{M}), s_{4}=\operatorname{Good}(\mathrm{G}), s_{5}=$ Very Good (VG), $s_{6}=$ Extremely Good (EG) $\}$ the extended linguistic term set is $\tilde{S}=\left\{s_{i} \mid i=0,1, \cdots, t\right\}$, with $s_{i}>s_{j}(i>j ; i, j \in\{0,1,2, \cdots, t\} t \geq 7)$ and the linguistic term $s_{i}(i \geq 7)$ has no practical semantics. The weight vector of criterion $C_{j}$ is $w=$ $\left(s_{4}, s_{2}, s_{1}, s_{3}\right)$. The assessment information of the five alternatives is to be evaluated using the linguistic term set $S_{1}$ by three decision-makers $D_{k}(k=1,2,3)$ whose associated weight vector is $\tau=\left(s_{4}, s_{3}, s_{2}\right)$. The subjective distances of the adjacent linguistic terms are $\operatorname{dist}\left(s_{m-1}, s_{m}\right)=1$ and the parameter $a=1.4$. The linguistic decision matrices are shown in $R_{1}, R_{2}$, and $R_{3}$ :

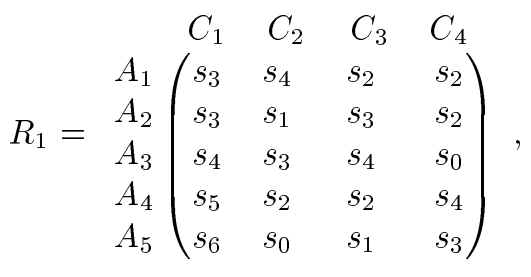



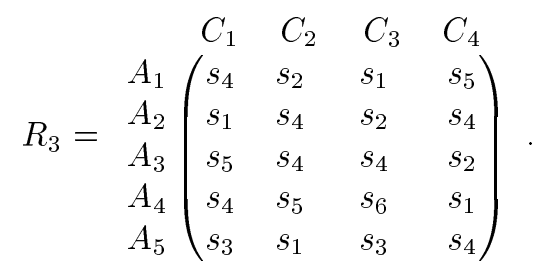

\subsection{The procedure for the 2-tuple linguistic MCGDM method}

To get the best alternative, the following steps are involved:

Step 1. Normalize the decision matrices.

The four criteria of the alternatives are regarded as the benefit-type criteria and the decision-makers provide the assessment information in the form of linguistic terms; therefore, the decision matrices $R_{k}=\left(h_{i j}^{k}\right)_{5 \times 4}\left(h_{i j}^{k} \in S_{1}, k=1,2,3\right)$ change nothing. The normalized decision matrices are denoted as $\bar{R}_{k}=R_{k}$;

Step 2. Convert the linguistic information into 2tuples.

Convert the matrix $\bar{R}_{k}=\left(h_{i j}^{k}\right)_{5 \times 4}$ to get the matrices $\tilde{R}_{1}, \tilde{R}_{2}$, and $\tilde{R}_{3}$ in form of 2-tuples. Similarly, convert the weight vector of decision-makers $\tau=$ $\left(s_{4}, s_{3}, s_{2}\right)$ to $\tilde{\tau}=\left(\left(s_{4}, 0\right),\left(s_{3}, 0\right),\left(s_{2}, 0\right)\right)$ and convert the weight vector of criteria $w=\left(s_{4}, s_{0}, s_{1}, s_{3}\right)$ to $\tilde{w}=\left(\left(s_{4}, 0\right),\left(s_{0}, 0\right),\left(s_{1}, 0\right),\left(s_{3}, 0\right)\right)$.

$$
\begin{aligned}
& \left.\begin{array}{ccccc}
C_{1} & C_{2} & C_{3} & C_{4} \\
\tilde{R}_{1}= & A_{1} \\
A_{2} & \left(s_{3}, 0\right) & \left(s_{4}, 0\right) & \left(s_{2}, 0\right) & \left(s_{2}, 0\right) \\
A_{3} & \left(s_{3}, 0\right) & \left(s_{1}, 0\right) & \left(s_{3}, 0\right) & \left(s_{2}, 0\right) \\
A_{4} & \left(s_{4}, 0\right) & \left(s_{3}, 0\right) & \left(s_{4}, 0\right) & \left(s_{0}, 0\right) \\
A_{5} & \left(s_{5}, 0\right) & \left(s_{2}, 0\right) & \left(s_{2}, 0\right) & \left(s_{4}, 0\right) \\
\left(s_{6}, 0\right) & \left(s_{0}, 0\right) & \left(s_{1}, 0\right) & \left(s_{3}, 0\right)
\end{array}\right),
\end{aligned}
$$

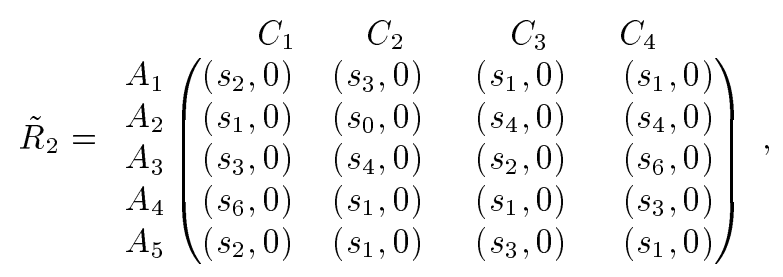

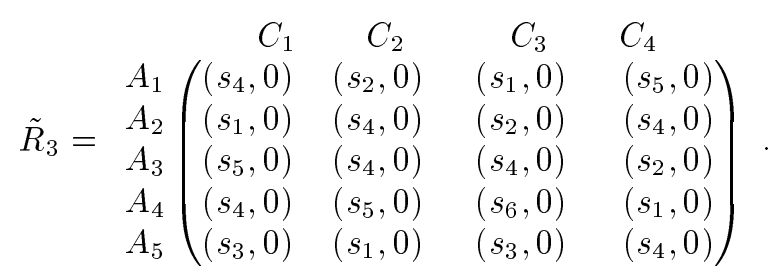

Step 3. Aggregate all the values of each alternative on each criterion to get the collective 2-tuple values.

Based on Definition 8 or Definition 10, the collective preference matrices can be obtained as the decision matrix $P$ by using the GE2T-WA operator and setting $\lambda=1$. Similarly, the decision matrix $\tilde{P}$ can be obtained by using the GE2T-WG operator as shown in Box II.

Step 4. Aggregate the collective preference matrices $P_{1}$ or $\tilde{P}$ to derive the overall values of the alternative $A_{i}$.

Based on Definition 9 or Definition 11, the overall preference value matrix can be obtained as 


\begin{tabular}{|c|c|c|c|c|c|c|}
\hline$P=$ & $\begin{array}{c}A_{1} \\
D_{1} \\
D_{2} \\
D_{3}\end{array}\left(\begin{array}{l}\left(s_{3},-0.225\right) \\
\left(s_{2},-0.295\right) \\
\left(s_{4},-0.442\right)\end{array}\right.$ & $\begin{array}{c}A_{2} \\
\left(s_{2}, 0.152\right) \\
\left(s_{2},-0.329\right) \\
\left(s_{2}, 0.486\right)\end{array}$ & $\begin{array}{c}A_{3} \\
\left(s_{2}, 0.195\right) \\
\left(s_{4}, 0.289\right) \\
\left(s_{4},-0.070\right)\end{array}$ & $\begin{array}{c}A_{4} \\
\left(s_{4}, 0.183\right) \\
\left(s_{4},-0.262\right) \\
\left(s_{4},-0.201\right)\end{array}$ & $\left.\begin{array}{c}A_{5} \\
\left(s_{3}, 0.302\right) \\
\left(s_{2},-0.462\right) \\
\left(s_{3},-0.279\right)\end{array}\right)$ & , \\
\hline$\tilde{P}=$ & $\begin{array}{c}A_{1} \\
D_{1} \\
D_{2} \\
D_{3}\end{array}\left(\begin{array}{c}\left(s_{7}, 0.082\right) \\
\left(s_{7},-0.198\right) \\
\left(s_{7}, 0.158\right)\end{array}\right.$ & $\begin{array}{c}A_{2} \\
\left(s_{7},-0.066\right) \\
\left(s_{6}, 0.031\right) \\
\left(s_{7}, 0.001\right)\end{array}$ & $\begin{array}{c}A_{3} \\
\left(s_{6}, 0.343\right) \\
\left(s_{7}, 0.304\right) \\
\left(s_{7}, 0.256\right)\end{array}$ & $\begin{array}{c}A_{4} \\
\left(s_{7}, 0.225\right) \\
\left(s_{7}, 0.118\right) \\
\left(s_{7}, 0.189\right)\end{array}$ & $\left.\begin{array}{c}A_{5} \\
\left(s_{6}, 0.349\right) \\
\left(s_{7},-0.251\right) \\
\left(s_{7}, 0.043\right)\end{array}\right)$ & \\
\hline
\end{tabular}

Box II

the decision matrices $T_{1}, T_{2}$, and $T_{3}$ by using the GE2T-OWA operator and setting $\lambda=1$. Similarly, the decision matrix $\tilde{T}$ can be obtained by using the GE2T-OWG operator:

$$
\begin{aligned}
& T=\begin{array}{ccc}
A_{1} & A_{2} & A_{3} \\
\left(\left(s_{3},-0.234\right)\right. & \left(s_{2}, 0.147\right) & \left(s_{4},-0.301\right)
\end{array} \\
& A_{4} \quad A_{5} \\
& \left.\left(s_{4},-0.211\right) \quad\left(s_{3},-0.421\right)\right)^{\prime} \\
& \tilde{T}=\begin{array}{ccc}
A_{1} & A_{2} & A_{3} \\
\left(\left(s_{8}, 0.021\right)\right. & \left(s_{8},-0.075\right) & \left(s_{8}, 0.028\right)
\end{array} \\
& A_{4} \quad A_{5} \\
& \left.\left(s_{8}, 0.066\right) \quad\left(s_{8},-0.066\right)\right)^{\circ}
\end{aligned}
$$

Step 5. Rank the alternatives and select the best one.

According to Definition 3, the ranking of the alternatives $A_{i}$ can be obtained, as shown in Table 2 .

The best alternative is $A_{4}$ that is obtained by using the GE2T-WA and GE2T-OWA operators and setting $\lambda=1$, or by using the GE2T-WG and GE2TOWG operators; however, the positions of $A_{1}$ and $A_{3}$ may swap.

The best alternative is always alternative $A_{4}$ that is obtained by using the GE2T-WG and GE2T-OWG operators and setting different values of $\lambda$; however, the ranking of the alternatives may be different, which is illustrated in Figure 1. Therefore, it is necessary for the decision-makers to set the values of $\lambda$ before aggregating the linguistic information. In general, a larger value of $\lambda$ may be set by more pessimistic decision-makers, which makes each alternative be associated with a higher objective numerical scale. By

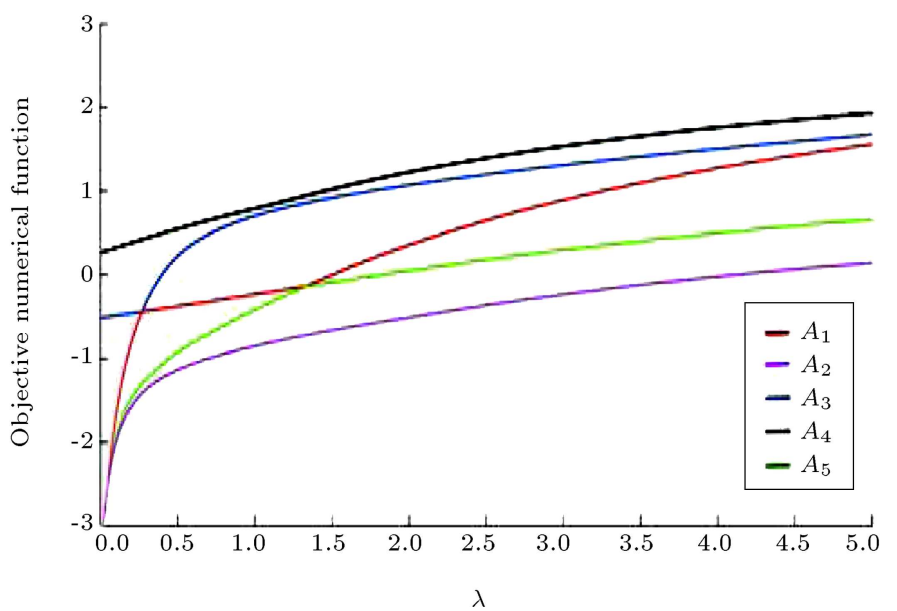

Figure 1. The ranking of the alternatives by setting different values of $\lambda$.

contrast, a smaller value of $\lambda$ may be set by more optimistic decision-makers. If the decision-makers do not give their preferences, the most commonly used value, $\lambda=1$, can be used.

\subsection{A comparison analysis and discussion}

In order to validate the feasibility of the proposed 2tuple linguistic MCGDM method, a comparative study is conducted with two other linguistic methods. The analysis is based on the same illustrative example and the methods used for comparison have been introduced in $[17-18]$.

1. When conducting the above illustrative example using the approach in [17], which is based on the Extended 2-Tuple Weighted Geometric (ET-WG) and the Extended 2-Tuple Ordered Weighted Geometric (ET-OWG) operators with 2-tuple linguistic information, the group of collective evaluation values for

Table 2. The ranking of the alternatives and the best alternative by using different operators and values of $\lambda$.

\begin{tabular}{llc}
\hline \multicolumn{1}{c}{ Operators } & Ranking results & The best alternative \\
\hline GE2T-WA and GE2T-OWA, $\lambda=1$ & $A_{4} \succ A_{3} \succ A_{1} \succ A_{5} \succ A_{2}$ & $A_{4}$ \\
GE2T-WG and GE2T-OWG & $A_{4} \succ A_{1} \succ A_{3} \succ A_{5} \succ A_{2}$ & $A_{4}$ \\
\hline
\end{tabular}




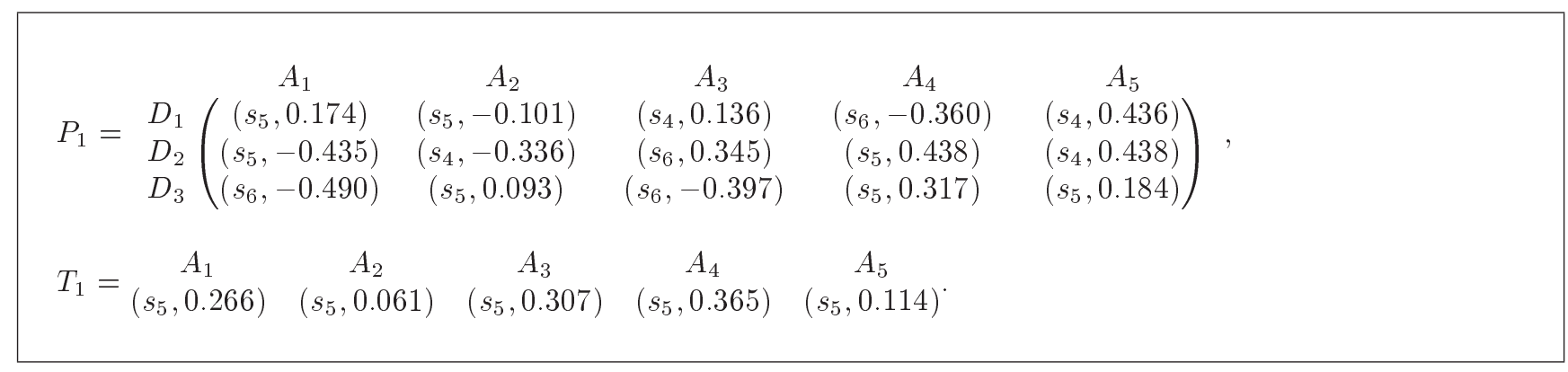

Box III

each alternative could be obtained and shown as matrix $P_{1}$ and the overall preference values matrix $T_{1}$ which is given in Box III.

The ranking of alternatives is $A_{4} \succ A_{3} \succ$ $A_{1} \succ A_{5} \succ A_{2}$ and the best alternative is $A_{4}$;

2. By applying the Generalized 2-Tuple Weighted Average (G-2TWA) and Generalized 2-Tuple Ordered Weighted Average (G-2TOWA) operators introduced in [18] to the same example, the aggregating result of the performance values of all decisionmakers is represented as the matrix $R^{\prime}$ and the overall preference values matrix $T_{2}$ as shown in Box IV. The ranking result is $A_{4} \succ A_{3} \succ A_{1} \succ$ $A_{5} \succ A_{2}$ and the best alternative is $A_{4}$.

Finally, the ranking results of the different methods can be shown in Table 3 .

From Table 3 , it can be seen that the best alternative obtained by applying the proposed approach is the same as the methods introduced in $[17,18]$. However, the criteria and experts' weights take the form of real numbers in the method proposed in [18]; thus, they cannot solve problems where the actual weights of the criteria and experts are difficult to provide using specific numbers. Alternatively, linguistic 2-tuples can accurately and easily deal with such a case. The method introduced in [17] only suits a situation where the linguistic terms are symmetrically distributed and distances of adjacent evaluation values are equal. This would degrade the feasibility of this method, especially when the linguistic terms are not uniformly and symmetrically distributed. Furthermore, it may also lose and/or distort the original information and make the final results be at odds with the actual decisionmaking.

Due to the fact that different kinds of operators were used, the ranking of the alternatives is not identical. When compared with the methods in $[17,18]$, the advantages of the proposed operators and approach are summarized as follows:

1. They can effectively deal with the evaluation information expressed by the linguistic variables and avoid losing and distorting the assessment infor-

$\left.\begin{array}{|ccccc|}\hline \multicolumn{6}{c}{C_{1}} & C_{2} & C_{3} & C_{4} \\ A_{1} & \left(s_{4},-0.111\right) & \left(s_{3},-0.111\right) & \left(s_{2},-0.333\right) & \left(s_{1}, 0.444\right) \\ R^{\prime}= & \left(s_{4},-0.444\right) & \left(s_{4},-0.444\right) & \left(s_{2},-0.333\right) & \left(s_{1},-0.333\right) \\ A_{3} & \left(s_{5},-0.111\right) & \left(s_{4}, 0\right) & \left(s_{3}, 0.222\right) & \left(s_{1}, 0.111\right) \\ A_{4} & \left(s_{6}, 0.444\right) & \left(s_{4},-0.111\right) & \left(s_{2}, 0.111\right) & \left(s_{1}, 0.444\right) \\ A_{5} & \left(s_{4},-0.444\right) & \left(s_{3},-0.333\right) & \left(s_{1}, 0.444\right) & \left(s_{1},-0.444\right)\end{array}\right)$,

Box IV

Table 3. The ranking results of the different methods.

\begin{tabular}{cll}
\hline Methods & \multicolumn{1}{c}{ Operators } & Ranking results \\
\hline The method in Reference [18] & G-2TWA and IG-2TOWA, $\lambda=1$ & $A_{4} \succ A_{3} \succ A_{1} \succ A_{5} \succ A_{2}$ \\
The method in Reference [17] & ET-WG and ET-OWG & $A_{4} \succ A_{3} \succ A_{1} \succ A_{5} \succ A_{2}$ \\
The proposed method & GE2T-WA and GE2T-OWA, $\lambda=1$ & $A_{4} \succ A_{3} \succ A_{1} \succ A_{5} \succ A_{2}$ \\
& GE2T-WG and GE2T-OWG & $A_{4} \succ A_{1} \succ A_{3} \succ A_{5} \succ A_{2}$ \\
\hline
\end{tabular}


mation by using the subjective sensation scale and objective numerical scale functions;

2. They remain feasible if the linguistic terms are not uniformly and symmetrically distributed;

3. They are suitable in situations where the semantics of linguistic terms and distances of adjacent linguistic terms are different and where the decisionmakers' sensations are changed.

\section{Conclusion}

The 2-tuple linguistic models for computing with words have made great progress in improving the accuracy and understanding of the processed results in decisionmaking. As they are a useful tool in processing linguistic information, many 2-tuple linguistic aggregation operators have been proposed. In order to improve the feasibility of 2-tuple linguistic models and overcome the existing limitations in 2-tuple linguistic aggregation operators, some new versions of 2-tuple linguistic aggregation operators have been developed in this paper. Firstly, the subjective sensation scale and objective numerical scale functions based on 2-tuple were proposed to resolve linguistic translation issues under different semantic and perceptual situations. Four new operators were proposed based on the scales: GE2T-WA operator, GE2T-OWA operator, GE2TWG operator, and GE2T-OWG operator. Furthermore, a new MCGDM method based on these operators under a linguistic environment was developed, which can avoid the information distortion caused by the transformation from non-equidistant distances of adjacent linguistic terms to linear equidistant numerical distances, reflect the differences between decision-makers' sensations, and remain feasible when the linguistic terms are not symmetrically distributed. Finally, the method was applied to a real case of choosing the best investment project in order to illustrate its potential applications; moreover, it was compared with other 2tuple linguistic methods to demonstrate its feasibility and effectiveness in solving linguistic MCGDM problems.

However, the method proposed in this paper only suits situations where the weights of the criterion and decision-makers are independent. In future research, the application of the developed operators will be extended to other domains and some 2-tuple linguistic methods will be proposed for situations where the weights of the criterion and decision-makers are not completely independent.

\section{Conflict of interests}

The authors declare that there is no conflict of interests regarding the publication of this paper.

\section{Acknowledgements}

This work was supported by the National Natural Science Foundation of China (Nos. 71271218, 71221061 and 71431006) and the Hunan Provincial Natural Science Foundation of China (No. 14JJ2009).

\section{References}

1. Delgado, M., Verdegay, J.L. and Vila, M.A. "Linguistic decision-making models", International Journal of Intelligent Systems, 7(5), pp. 479-492 (1992).

2. Herrera, F. and Herrera-Viedma, E. "Linguistic decision analysis: steps for solving decision problems under linguistic information", Fuzzy Sets and Systems, 115(1), pp. 67-82 (2000).

3. Wang, J.Q., Yu, S.M., Wang, J., Chen, Q.H., Zhang, H.Y. and Chen, X.H. "An interval type-2 fuzzy number based approach for multi-criteria group decision-making problems", International Journal of Uncertainty, Fuzziness and Knowledge-Based Systems, 23(4), pp. 1-17 (2015).

4. Fu, S., Liu, Z.L., Zhou, H.J., Song, D. and Xiao, Y.Z. "Trapezoidal fuzzy number attitude indicators group decision making approaches based on fuzzy language", Journal of Applied Sciences, 14(19), pp. 2304-2308 (2014).

5. Wang, X.F., Wang, J.Q. and Yang, W.E. "Multicriteria group decision making method based on intuitionistic linguistic aggregation operators", Journal of Intelligent and Fuzzy Systems, 26(1), pp. 115-125 (2014).

6. Yager, R.R. "An approach to ordinal decision making", International Journal of Approximate Reasoning, 12(3), pp. 237-261 (1995).

7. Rodríguez, R.M. and Martinez, L. "An analysis of symbolic linguistic computing models in decision making", International Journal of General Systems, 42(1), pp. 121-136 (2013).

8. Chin, K.S. and Fu, C. "Integrated evidential reasoning approach in the presence of cardinal and ordinal preferences and its applications in software selection", Expert Systems with Applications, 44, pp. 6718-6727 (2014).

9. Yuen, K.K.F. "Combining compound linguistic ordinal scale and cognitive pairwise comparison in the rectified fuzzy TOPSIS method for group decision making", Fuzzy Optimization and Decision Making, 13(1), pp. 105-130 (2014).

10. Yang, W.E. and Wang, J.Q. "Multi-criteria semantic dominance: A linguistic decision aiding technique based on incomplete preference information", European Journal of Operational Research, 231(1), pp. 171181 (2013).

11. Wang, J., Wang, J.Q., Zhang, H.Y. and Chen, X.H. "Multi-criteria decision-making based on hesitant fuzzy linguistic term sets: an outranking 
approach", Knowledge-Based Systems (2015). DOI: 10.1016/j.knosys.2015.06.007

12. Wang, G.Y., Xu, C.L., Zhang, Q.H. and Wang, X.R. "A multi-step backward cloud generator algorithm", Lecture Notes in Computer Science, 7413, pp. 313-322 (2012).

13. Wang, J.Q., Wang, P., Zhang, H.Y. and Chen, X.H. "Atanassov's interval-valued intuitionistic linguistic multi-criteria group decision-making method based on trapezium cloud model", IEEE Transaction on Fuzzy Systems, 23(3), pp. 542-554 (2015).

14. Wang, J.Q., Peng, L., Zhang, H.Y. and Chen, X.H. "Method of multi-criteria group decision-making based on cloud aggregation operators with linguistic information", Information Sciences, 274, pp. 177-191 (2014).

15. Wang, J.Q., Peng, J.J., Zhang, H.Y., Liu, T. and Chen, X.H. "An uncertain linguistic multi-criteria group decision-making method based on a cloud model", Group Decision and Negotiation, 24(1), pp. 171-192 (2015).

16. Herrera, F. and Martinez, L. "A 2-tuple fuzzy linguistic representation model for computing with words", IEEE Transactions on Fuzzy Systems, 8(6), pp. 746752 (2000).

17. Wei, G.W. "A method for multiple attribute group decision making based on the ET-WG and ET-OWG operators with 2-tuple linguistic information", $E x$ pert Systems with Applications, 37(12), pp. 7895-7900 (2010).

18. Wei, G.W. "Some generalized aggregating operators with linguistic information and their application to multiple attribute group decision making", Computers and Industrial Engineering, 61(1), pp. 32-38 (2011).

19. Wei, G.W. and Zhao, X.F. "Some dependent aggregation operators with 2-tuple linguistic information and their application to multiple attribute group decision making", Expert Systems with Applications, 39(5), pp. 5881-5886 (2012).

20. Herrera, F. "An overview on the 2-tuple linguistic model for computing with words in decision making: extensions, applications and challenges", Information Sciences, 207, pp. 1-18 (2012).

21. Herrera, F. and Martinez, L. "An approach for combining linguistic and numerical information based on the 2-tuple fuzzy linguistic representation model in decision-making", International Journal of Uncertainty, Fuzziness and Knowledge-Based Systems, 8(5), pp. 539-562 (2000).

22. Herrera, F., Martinez, L. and Sánchez, P.J. "Managing non-homogeneous information in group decision making", European Journal of Operational Research, 166(1), pp. 115-132 (2005).

23. Estrella, F.J., Espinilla, M., Herrera, F. and Martinez, L. "FLINTSTONES: A fuzzy linguistic decision tools enhancement suite based on the 2-tuple linguistic model and extensions", Information Sciences, 280, pp. 152-170 (2014).

24. Herrera, F. and Martinez, L. "A model based on linguistic 2-tuples for dealing with multigranular hierarchical linguistic contexts in multi-expert decisionmaking", IEEE Transactions on Systems, Man, and Cybernetics, 31(2), pp. 227-234 (2001).

25. Liu, H.B. and Cai, J.F. "A method for group decision making with multi-granularity interval reciprocal linguistic judgment matrix", IEEE International Conference on Computer and Management, pp. 1-4 (2011).

26. Dong, Y.C., Li, C.C., Xu, Y.F. and Gu, X. "Consensus-based group decision making under multigranular unbalanced 2-tuple linguistic preference relations", Group Decision and Negotiation, 24(2), pp. 217-242 (2014)

27. Morente-Molinera, J.A., Pérez, I.J., Ureña, R. and Herrera-Viedma, E. "On multi-granular fuzzy linguistic modelling in group decision making problems: a systematic review and future trends", KnowledgeBased Systems, 74, pp. 49-60 (2015).

28. Herrera, F., Herrera-Viedma, E. and Martinez, L. "A fuzzy linguistic methodology to deal with unbalanced linguistic term sets", IEEE Transactions on Fuzzy Systems, 16(2), pp. 354-370 (2008).

29. Jiang, L., Liu, H.B. and Cai, J.F. "The power average operator for unbalanced linguistic term sets", Information Fusion, 22, pp. 85-94 (2015).

30. Meng, D. and Pei, Z. "On weighted unbalanced linguistic aggregation operators in group decision making", Information Sciences, 223, pp. 31-41 (2013).

31. Dong, Y.C., Li, C.C. and Herrera, F. "An optimization-based approach to adjusting unbalanced linguistic preference relations to obtain a required consistency level", Information Sciences, 292, pp. 2738 (2015).

32. Wang, J.H. and Hao, J.Y. "A new version of 2-tuple fuzzy linguistic representation model for computing with words", IEEE Transactions on Fuzzy Systems, 14(3), pp. 435-445 (2006).

33. Li, C.C. and Dong, Y.C. "Multi-attribute group decision making methods with proportional 2-tuple linguistic assessments and weights", International Journal of Computational Intelligence Systems, 7, pp. 758-770 (2014).

34. Dong, Y.C., Xu, Y.F. and Yu, S. "Computing the numerical scale of the linguistic term set for the 2tuple fuzzy linguistic representation model", IEEE Transactions on Fuzzy Systems, 17(6), pp. 1366-1378 (2009).

35. Dong, Y.C., Zhang, G.Q., Hong, W.C. and Yu, S. "Linguistic computational model based on 2-tuples and intervals", IEEE Transactions on Fuzzy Systems, 21(6), pp. 1006-1018 (2013). 
36. Dong, Y.C., Li, C.C. and Herrera, F. "Connecting the numerical scale model to the unbalanced linguistic term sets", IEEE International Conference on Fuzzy Systems, pp. 455-462 (2014).

37. Dong, Y.C., Hong, W.C., Xu, Y.F. and Yu, S. "Numerical scales generated individually for analytic hierarchy process", European Journal of Operational Research, 229(3), pp. 654-662 (2013).

38. Dong, Y.C. and Herrera-Viedma, E. "Consistencydriven automatic methodology to set interval numerical scales of 2-tuple linguistic term sets and its use in the linguistic GDM with preference relation", IEEE Transactions on Cybernetics, 45(4), pp. 780-792 (2015).

39. Merigó, J.M. and Gil-Lafuente, A.M. "Induced 2-tuple linguistic generalized aggregation operators and their application in decision-making", Information Sciences, 236, pp. 1-16 (2013).

40. Li, C.G., Zeng, S.Z., Pan, T.J. and Zheng, L.N. "A method based on induced aggregation operators and distance measures to multiple attribute decision making under 2-tuple linguistic environment", Journal of Computer and System Sciences, 80(7), pp. 13391349 (2014).

41. Tao, Z.F., Chen, H.Y., Zhou, L.G. and Liu, J.P. "On new operational laws of 2-tuple linguistic information using Archimedean t-norm and s-norm", KnowledgeBased Systems, 66, pp. 156-165 (2014).

42. Zhang, H.M. "Some interval-valued 2-tuple linguistic aggregation operators and application in multiattribute group decision making", Applied Mathematical Modelling, 37(6), pp. 4269-4282 (2013).

43. Zhang, Z.M. and Wu, C. "2-tuple linguistic Bonferroni mean operators and their application to multiple attribute group decision making", British Journal of Mathematics and Computer Science, 4(11), pp. 15671614 (2014).

44. Wei, G.W. "Some harmonic aggregation operators with 2-tuple linguistic assessment information and their application to multiple attribute group decision making", International Journal of Uncertainty, Fuzziness and Knowledge-Based Systems, 19(06), pp. 977998 (2011).

45. Wang, H.J., Zhao, X.F., Lin, R. and Wei, G.W. "Some extended harmonic aggregation operators with 2-tuple linguistic information and their application to potential evaluation of emerging technology commercialization", JDCTA: International Journal of Digital Content Technology and its Applications, 7(2), pp. 668676 (2013).

46. Wan, S.P. "2-Tuple linguistic hybrid arithmetic aggregation operators and application to multi-attribute group decision making", Knowledge-Based Systems, 45, pp. 31-40 (2013).

47. Xu, Y.J., Shi, P.F., Merigó, J.M. and Wang, H.M. "Some proportional 2-tuple geometric aggregation operators for linguistic decision making", Journal of
Intelligent and Fuzzy Systems, 25(3), pp. 833-843 (2013).

48. Xu, Y.J. and Wang, H.M. "Approaches based on 2tuple linguistic power aggregation operators for multiple attribute group decision making under linguistic environment", Applied Soft Computing, 11(5), pp. 3988-3997 (2011).

49. Liu, H.C., Lin, Q.L. and Wu, J. "Dependent interval 2-tuple linguistic aggregation operators and their application to multiple attribute group decision making", International Journal of Uncertainty, Fuzziness and Knowledge-Based Systems, 22(5), pp. 717-735 (2014).

50. Wang, J.Q., Wang, D.D., Zhang, H.Y. and Chen, X.H. "Multi-criteria group decision making method based on interval 2-tuple linguistic information and Choquet integral aggregation operators", Soft Computing, 19(2), pp. 389-405 (2015).

51. Xu, Z.S. "A method based on linguistic aggregation operators for group decision making with linguistic preference relations", Information Sciences, 166(1), pp. 19-30 (2004).

52. Wang, J.Q., Wu, J.T., Wang, J., Zhang, H.Y. and Chen, X.H. "Interval-valued hesitant fuzzy linguistic sets and their applications in multi-criteria decisionmaking problems", Information Sciences, 288, pp. 5572 (2014).

53. Wang, J., Wang, J.Q, Zhang, H.K. and Chen, X.H. "Multi-criteria group decision-making approach based on 2-tuple linguistic aggregation operators with multihesitant fuzzy linguistic information", International Journal of Fuzzy Systems, 18(1), pp. 81-97 (2016).

\section{Biographies}

Yin-xiang Ma was born in China, in 1990. He is a Master degree candidate in Management Science and Engineering at Central South University, Changsha, China. His research interests focus on multi-attribute group decision-making method and aggregation operators.

Jing Wang received the MSc degree in Information Engineering from the University of Osnabrueck, Osnabrueck, Germany, in 2006. She is currently working toward the $\mathrm{PhD}$ degree in the School of Business, Central South University, Changsha, China. She is also a Lecturer at International College, Central South University of Forestry and Technology. Her current research interests include decision-making theory and application, risk management and control, and information management.

Jian-qiang Wang received the $\mathrm{PhD}$ degree in Management Science and Engineering from Central South University, Changsha, China, in 2005. He is currently a Professor in the School of Business, Central South Uni- 
versity. His current research interests include decisionmaking theory and application, risk management and control, and information management.

Xiao-hong Chen received the $\mathrm{PhD}$ degree in Management from the Tokyo University of Technology, Tokyo, Japan, in 1999. She is currently a Professor in the
School of Business, Central South University, Changsha, China. Her current research interests include decision theory and methods, decision support systems, resource-saving, and the environment-friendly society. She has published academic papers in many journals, including decision support system, expert systems with applications, etc. 